\title{
Uniform interpolation and sequent calculi in modal logic
}

\author{
Rosalie Iemhoff ${ }^{1}$
}

Received: 28 March 2015 / Accepted: 2 May 2018 / Published online: 10 May 2018

(C) The Author(s) 2018

\begin{abstract}
A method is presented that connects the existence of uniform interpolants to the existence of certain sequent calculi. This method is applied to several modal logics and is shown to cover known results from the literature, such as the existence of uniform interpolants for the modal logic K. New is the result that KD has uniform interpolation. The results imply that for modal logics K4 and S4, which are known not to have uniform interpolation, certain sequent calculi cannot exist.
\end{abstract}

Keywords Uniform interpolation · Sequent calculus · Modal logic $\cdot$ Propositional quantifiers

Mathematics Subject Classification 03B05 -03B45 - 03F03

\section{Introduction}

Interpolation has been studied in a variety of settings since William Craig proved in 1957 that classical predicate logic has interpolation. Interpolation is a desirable property for a logic to have because it indicates that the logic is well-behaved in a sense reminiscent to analyticity: if an implication $\varphi \rightarrow \psi$ holds in the logic, then there is a $\chi$ in the common language of $\varphi$ and $\psi$ that interpolates, that is, such that $\varphi \rightarrow \chi$ and $\chi \rightarrow \psi$ hold. What the common language is depends on the logic one considers. In propositional logics it typically means that all atoms in $\chi$ occur in both $\varphi$ and $\psi$.

Rosalie Iemhoff

r.iemhoff@uu.nl

1 Department of Philosophy, Utrecht University, Janskerkhof 13, 3512 BL Utrecht, The Netherlands 
In 1992 it was proved by Andrew Pitts that intuitionistic propositional logic IPC, which has interpolation, also satisfies the stronger property of uniform interpolation [6]: given a formula $\varphi$ and an atom $p$, there exist uniform interpolants $\forall p \varphi$ and $\exists p \varphi$ which are formulas (in the language of IPC) that do not contain $p$ and such that for all $\psi$ not containing $p$ :

$$
\vdash \varphi \rightarrow \psi \Leftrightarrow \vdash \exists p \varphi \rightarrow \psi \quad \vdash \psi \rightarrow \varphi \Leftrightarrow \vdash \psi \rightarrow \forall p \varphi .
$$

This is a strengthening of interpolation in which the interpolant only depends on the premiss (in the case of $\exists$ ) or the conclusion (in the case of $\forall$ ) of the given implication: $\forall p_{1} \ldots \forall p_{n} \varphi$ and $\exists p_{1} \ldots \exists p_{n} \varphi$ interpolate any $\psi \rightarrow \varphi$ and $\varphi \rightarrow \psi$, respectively, such that $p_{1}, \ldots, p_{n}$ do not occur in $\psi$. In case $\forall p_{1} \ldots \forall p_{n} \varphi$ or $\exists p_{1} \ldots \exists p_{n} \varphi$ contain additional variables not in the common language of $\varphi$ and $\psi$, these have to be replaced by $\top$ or $\perp$ or a variable in the common language.

As the notation suggests, the fact that the uniform interpolants are definable in IPC also shows that the propositional quantifiers are definable in that logic. From the algebraic point of view the quantifiers are left and right adjoints of certain embeddings.

Around the time that Pitts obtained his result, Shavrukov proved, by completely different methods, that the modal logic GL has uniform interpolation [7]. Since then, uniform interpolation has been established for various other logics, including the modal logics K and KT [1,9,10]. Intriguingly, the modal logics K4 and S4 do not have uniform interpolation $[1,3]$. As there are only seven propositional intermediate logics with interpolation [5], the number of intermediate logics with uniform interpolation is necessarily bounded by that number. Ghilardi and Zawadowski showed that there are exactly that many [4].

Whereas in the presence of a decent analytic sequent calculus, proofs of interpolation are often relatively straightforward, proofs of uniform interpolation are in general quite complex. Moreover, it is less clear in how far, if at all, proof systems such as sequent calculi can be of help in establishing the property, as there are logics with analytic sequent calculi that have uniform interpolation ( $\mathrm{K}$ and $\mathrm{GL}$ ) as well as logics with analytic sequent calculi that do not (K4 and S4).

In this paper our aim is twofold: to develop a method to extract uniform interpolants from sequent calculi and to prove, using this method, that logics without uniform interpolation lack certain calculi. For both aims it holds that the more general the considered calculi are, the stronger the result. In this paper we restrict ourselves to classical propositional modal logics, but the method applies to intermediate logics as well. For such logics the method is more complicated though, since $\exists$ is not expressible in terms of $\forall$, whereas in the classical case one can just take $\neg \forall p \neg$ for $\exists p$. We plan to treat intermediate logics in a separate paper.

Our construction of uniform interpolants on the basis of calculi provides a modular approach to uniform interpolation. Namely, to establish that the extension of a given calculus with uniform interpolation by a given rule still has uniform interpolation, only certain local properties have to be verified, whereas for most other methods the proof of uniform interpolation for the extension has to be given again in full. Our method is different from but inspired by Pitts' ingenious syntactic method. Bílková used a similar method as Pitts in combination with a clever termination strategy for 
the logics K, GL, KT and Grz [1]. Most other proofs of uniform interpolation are of a semantical nature.

In our method, we isolate a certain type of propositional rules called focussed rules and a certain type of modal rules called focussed modal rules and prove that any logic with a terminating balanced sequent calculus consisting of focussed and focussed modal rules has uniform interpolation. Termination means that in no rule the premisses are more complex, in a certain given ordering, than the conclusion. And a calculus is balanced if for certain combinations of left and right rules, either both rules belong to the calculus or both do not. This result then implies the well-known fact that classical propositional logic has uniform interpolation, and that so do $\mathrm{K}$ and $\mathrm{KD}$. Whereas for $\mathrm{K}$ this was already known, for KD the result is new. Our results also imply that K4 and S4 cannot have sequent calculi of the above kind. Although for S4 this might be easy to infer in another way, for K4 this seems to be a novel insight.

Besides the mentioned results, uniform interpolation is obtained for various other modal logics as well. The main interest in these results lies not so much in the logics involved, but rather in the illustration they provide of the flexibility of the method developed here. The calculi covered in this paper are not the only calculi to which our method applies, or so we conjecture. It seems likely that similar reasoning applies to other calculi for modal and intermediate logics. We chose, however, to first set up the general framework in this paper because we think it is of interest in itself, and in this way we steer clear of the complexities that might arise once the existential quantifier is no longer expressible in terms of the universal quantifier, as is the case in nonclassical logics.

\section{Logics and calculi}

The logics we consider are modal propositional logics, formulated in a language $\mathcal{L}$ that contains constants $\top$ and $\perp$, propositional variables or atoms $p, q, r, \ldots$ and the connectives $\wedge, \vee, \neg, \rightarrow$ and the modal operator $\square$. We assume that all logics we consider are extensions of classical propositional logic CPC and satisfy the necessitation rule, but we do not assume them to be normal. The logics are given by consequence relations denoted by $\vdash$ or $\vdash_{\mathrm{L}}$ and we assume them to be consistent, meaning to not derive $\perp$. $\mathcal{F}$ denotes the set of formulas in $\mathcal{L}$ and $\mathcal{M}$ is the set of all finite multisets of formulas in $\mathcal{F}$. Given a set of atoms $\mathcal{P}, \mathcal{F}(\mathcal{P})$ denotes all formulas in $\mathcal{L}$ in which all atoms belong to $\mathcal{P}$. The language $\mathcal{L}_{\text {qf }}$ is defined to be the extension of $\mathcal{L}$ with propositional quantifiers $\forall p$ and $\exists p$ for every atom $p$, and $\mathcal{F}_{\text {qf }}$ is the set of formulas in that language.

Sequents are expressions $\Gamma \Rightarrow \Delta$, where $\Gamma$ and $\Delta$ are finite multisets of formulas, that are interpreted as $I(\Gamma \Rightarrow \Delta)=(\bigwedge \Gamma \rightarrow \bigvee \Delta)$, where $\bigwedge \varnothing$ and $\bigvee \varnothing$ are interpreted as $\top$ and $\perp$, respectively. We denote finite multisets by $\Gamma, \Pi, \Delta, \Sigma$. In a sequent, notation $\Pi, \Gamma$ is short for $\Gamma \cup \Pi$. We also define ( $a$ for antecedent, $s$ for succedent):

$$
(\Gamma \Rightarrow \Delta)^{a} \equiv_{\text {def }} \Gamma \quad(\Gamma \Rightarrow \Delta)^{s} \equiv_{\text {def }} \Delta
$$


For sequents $S$ and $S^{\prime}, S \subseteq S^{\prime}$ denotes that $S^{a} \subseteq S^{\prime a}$ and $S^{S} \subseteq S^{\prime s}$. When sequents are used in the setting of formulas, we often write $S$ for $I(S)$, such as in $\vdash \bigvee_{i}\left(S_{i} \Rightarrow S\right)$, which thus means $\vdash \bigvee_{i}\left(I\left(S_{i}\right) \rightarrow I(S)\right)$. Multiplication of sequents is defined as

$$
S_{1} \cdot S_{2} \equiv_{\text {def }}\left(S_{1}^{a} \cup S_{2}^{a} \Rightarrow S_{1}^{s} \cup S_{2}^{s}\right) .
$$

The set $\mathcal{F}_{\text {ex }}$ is the smallest set of expressions that contains $\mathcal{F}$, is closed under the connectives and modal operator, and if $S$ is a sequent such that all its elements belong to $\mathcal{F}$, then $\forall p S$ and $\exists p S$ belong to $\mathcal{F}_{\text {ex }}$. For example, $\square \forall p_{1}\left(p_{1} \Rightarrow p_{2}\right)$ belongs to $\mathcal{F}_{\text {ex }}$, but $\forall p_{1} \forall p_{2}\left(p_{1} \Rightarrow p_{2}\right)$ does not. We say that a sequent is in $\mathcal{L}$ or in $\mathcal{F}$ if all formulas that occur in it are in $\mathcal{F}$, and likewise for $\mathcal{L}_{\text {qf }}, \mathcal{F}_{\text {qf }}$ and $\mathcal{F}_{\text {ex }}$.

The interpretation of $\mathcal{F}_{\text {ex }}$ into $\mathcal{F}_{\text {qf }}$ is the identity on formulas in $\mathcal{F}$, commutes with the logical operators and interprets quantified sequents as

$$
\forall p S \equiv_{d e f} \forall p I(S)
$$

\subsection{Rules and axioms}

For a proper syntactic treatment of interpolation we need to make a distinction between the language and the meta-language. $\overline{\mathcal{L}}$ is a copy of $\mathcal{L}$ in which every atom $p$ is replaced by $\bar{p}$. The set $\overline{\mathcal{F}}$ of formulas in this language is defined as usual. $\overline{\mathcal{M}}$ is an infinite set of symbols for meta-multisets, the elements we denote by $\bar{\Gamma}, \bar{\Pi}, \bar{\Delta}, \bar{\Sigma}$. A meta-sequent is an expression $X \Rightarrow Y$, where $X$ and $Y$ are multisets consisting of elements in $\overline{\mathcal{F}} \cup \overline{\mathcal{M}}$.

A substitution $\sigma$ is a map from $\overline{\mathcal{F}} \cup \overline{\mathcal{M}}$ to $\mathcal{F} \cup \mathcal{M}$ that commutes with the connectives and modal operator and such that $\sigma[\overline{\mathcal{F}}] \subseteq \mathcal{F}$ and $\sigma[\overline{\mathcal{M}}] \subseteq \mathcal{M}$. Sub is the set of all substitutions.

A sequent calculus is a set of rules, which are expressions of the form

$$
\frac{S_{1} \quad S_{2} \quad \ldots \quad S_{n}}{S_{0}} \mathcal{R}
$$

for some meta-sequents $S_{0}, S_{1}, \ldots, S_{n}$. A rule is also called an axiom in case there are no premisses, so that it consists of a single meta-sequent. Thus axioms are considered to be special instances of rules.

For any substitution $\sigma$, the inference

$$
\frac{\sigma S_{1} \quad \sigma S_{2} \quad \ldots \quad \sigma S_{n}}{\sigma S_{0}} \sigma \mathcal{R}
$$

is an instance of $\mathcal{R}$. Throughout this paper we denote schematic rules by $\mathcal{R}$ and instances of rules by $R$. Sets of rules are denoted by $\mathcal{R}$, and $\mathcal{R}_{\text {ins }}$ denotes the set of instances of rules in $\mathcal{R}$. We use the same symbols for axioms but it will always be clear from the context, explicitly stated or immaterial whether the rule is an axiom or not.

As is often done implicitly in papers on sequent calculi, we will from now on confuse the meta-level with the object-level by omitting overscores and the word 
"meta", trusting that it will always be clear from the context (or does not matter) on which level we are. For example, an axiom such as $\bar{\Gamma}, \bar{p} \Rightarrow \bar{p}, \bar{\Delta}$ will simply be written as $\Gamma, \varphi \Rightarrow \varphi, \Delta$.

Sometimes rules come with side conditions, which formally are conditions on the substitutions that are allowed. For example, an axiom $\Gamma, \varphi \Rightarrow \varphi, \Delta$ could have the side condition that for $\varphi$ only atoms may be substituted. Also in this case we follow common procedure, by writing such an axiom as $\Gamma, p \Rightarrow p, \Delta$.

For $\mathcal{R}$ as in (1) and for any meta-sequent $S, \mathcal{R}(S)$ denotes the rule

$$
\frac{S \cdot S_{1} \quad S \cdot S_{2} \ldots S \cdot S \cdot S_{n}}{S \cdot S_{0}} \mathcal{R}(S)
$$

For instances $R$ and sequents $S, R(S)$ is defined similarly.

A rule is backwards applicable to a sequent $S$ when there is at least one instance of the rule with $S$ as the conclusion. An instance of a rule is backwards applicable to $S$ if its conclusion is $S$. A sequent is free if it is not the conclusion of any instance of any rule and it is not an instance of any axiom.

When a logic $L$ given by a consequence relation $\vdash_{L}$ has a sequent calculus with respect to which it is sound and complete, then we assume that the consequence relation is such that for every instance $S_{1} \ldots S_{n} / S_{0}$ of a rule in the calculus, $I\left(S_{1}\right), \ldots, I\left(S_{n}\right) \vdash_{\mathrm{L}} I\left(S_{0}\right)$ holds.

By $\vdash_{\mathrm{CPC}}^{\mathcal{R}}$ we denote the smallest consequence relation containing $\mathcal{R}$ and such that $\varphi_{1}, \ldots, \varphi_{n} \vdash_{\mathrm{CPC}}^{\mathcal{R}} \psi$ holds whenever $\psi$ or $\left(\bigwedge \varphi_{i} \rightarrow \psi\right)$ hold in CPC.

\subsection{Focussed rules}

A rule $\mathcal{R}$ that is not an axiom is focussed if there are meta-sequents $S_{1}, \ldots, S_{n}$, a meta-sequent $S_{0}$ consisting of exactly one meta-formula that is not an atom, and a meta-sequent $S=(\Gamma \Rightarrow \Delta)$ for two distinct meta-multisets $\Gamma$ and $\Delta$ that do not occur in the $S_{i}$, such that $\mathcal{R}$ is the rule

$$
\frac{S \cdot S_{1} \quad S \cdot S_{2} \quad \ldots \quad S \cdot S_{n}}{S \cdot S_{0}} \mathcal{R}
$$

The rule is a right rule in case $S_{0}^{a}$ is empty and a left rule otherwise.

An axiom is focussed if it is either of the form $(\Gamma, r \Rightarrow r, \Delta)$ or $(\Gamma, \perp \Rightarrow \Delta)$ or $(\Gamma \Rightarrow \top, \Delta)$. Thus when we speak of a focussed rule, this either means a focussed rule that is not an axiom, or a focussed axiom. A calculus is focussed if every rule in it is focussed.

Typical focussed rules are the left and right rules of many Gentzen calculi. The right conjunction rule

$$
\frac{\Gamma \Rightarrow \varphi, \Delta \quad \Gamma \Rightarrow \psi, \Delta}{\Gamma \Rightarrow \varphi \wedge \psi, \Delta}
$$


is clearly focussed, as one can take $(\Gamma \Rightarrow \Delta)$ for $S,(\Rightarrow \varphi \wedge \psi)$ for $S_{0}$ and $(\Rightarrow \varphi)$ and $(\Rightarrow \psi)$ for $S_{1}$ and $S_{2}$, respectively.

\subsection{Terminating calculi}

A calculus is defined to be a collection of axioms and rules. If $\mathrm{G}$ is a calculus, $\vdash_{\mathrm{G}} S$ denotes that sequent $S$ is derivable in $\mathrm{G}$. $\mathrm{G}$ is closed under weakening if whenever a sequent $S$ is derivable, the sequent $S \cdot S^{\prime}$ is derivable for any sequent $S^{\prime}$. It is terminating if it is finite and for all sequents $S$ and rules in the calculus there are at most finitely many instances of the rule backwards applicable to $S$, and there is a well-founded order $\prec$ on sequents such that in every rule of the calculus the premisses come before the conclusion in this order, all proper subsequents of a sequent come before that sequent, $(\Gamma \Rightarrow) \prec(\square \Gamma \Rightarrow)$ and $(\Rightarrow \Gamma) \prec(\Rightarrow \square \Gamma)$ for any multiset $\Gamma$, and no sequent comes before the empty sequent.

A typical example of a rule that in general cannot belong to a terminating calculus is the cut rule, as there can be infinitely many instances of the rule with the same conclusion. We will see that many standard cut-free calculi for modal logic are terminating.

All calculi in this paper are assumed to derive at least all sequents for which the interpretation holds in classical propositional logic.

\subsection{Partitions}

The method to construct uniform interpolants that we are going to develop uses partitions of sequents and rules. A partition of a sequent $S$ is an ordered pair $\left(S^{r}, S^{i}\right)(i$ for interpolant, $r$ for rest) such that $S=S^{r} \cdot S^{i}$, where = denotes equality on multisets. It is a p-partition if $p$ does not occur in $S^{r}$. It is trivial if $S^{r}$ is the empty sequent, and whence $S^{i}=S$.

A $(p$-)partition $\operatorname{pt}(\mathcal{R})$ of a rule

$$
\frac{S_{1} \quad \ldots \quad S_{n}}{S_{0}} \mathcal{R}
$$

is an expression of the form

$$
\frac{S_{1}^{r} \cdot S_{1}^{i} \ldots S_{n}^{r} \cdot S_{n}^{i}}{S_{0}^{r} \cdot S_{0}^{i}} \operatorname{pt}(\mathcal{R})
$$

where $\left(S_{j}^{r}, S_{j}^{i}\right)$ is a $\left(p\right.$-)partition of $S_{j}$. The $\left(p\right.$-)partitions $\left(S_{1}^{r}, S_{1}^{i}\right), \ldots,\left(S_{n}^{r}, S_{n}^{i}\right)$ of the premisses of $\mathcal{R}$ are said to $\mathcal{R}$-correspond to $\left(S_{0}^{r}, S_{0}^{i}\right)$ under pt.

The way the partition of a rule is denoted above is slightly ambiguous. Although $S \cdot S^{\prime}$ is equal to $S^{\prime} \cdot S$ as a sequent, in denoting the partition of a rule as is done above, $S \cdot S^{\prime}$ refers to the partition $\left(S, S^{\prime}\right)$ of sequent $S \cdot S^{\prime}$, while $S^{\prime} \cdot S$ refers to the partition 
$\left(S^{\prime}, S\right)$ of the same sequent $S \cdot S^{\prime}$. We allow this ambiguity in order to keep the notation of partitions of rules simple.

For $\star \in\{i, r\}$ the rule $\mathcal{R}^{\star}$ is defined as

$$
\frac{S_{1}^{\star} \quad \ldots \quad S_{n}^{\star}}{S_{0}^{\star}} \mathcal{R}^{\star}
$$

Note that for every $p$-partition of $S_{0}$ there exists at least one $p$-partition of $\mathcal{R}$ that partitions $S_{0}$ in exactly the same way, for example by choosing for the $S_{i}^{r}(i>0)$ the empty sequents.

\subsection{Rank}

Given an order $\prec$ on sequents we define another order, the rank, on $\mathcal{F}_{\text {ex }}$ as follows. We first associate the following set of formulas with a formula. Given a formula $\varphi$ in $\mathcal{F}_{\text {ex }}$, let $\mathrm{qf}(\varphi)$ be the multiset consisting of all occurrences of subformulas of the form $Q p S$ in $\varphi$. The order on multisets of the form $q f(\varphi)$ is in the style of [2]: qf $(\varphi) \prec_{\text {qf }}$ qf $(\psi)$ iff $\operatorname{qf}(\varphi)$ is the result of replacing one or more formulas of the form $Q p S$ in $\mathrm{qf}(\psi)$ by zero or more formulas of the form $Q q S^{\prime}$ with $S^{\prime} \prec S$. This order is well-defined since by definition such $S$ are sequents in $\mathcal{L}$, to which $\prec$ applies.

The order on $\mathcal{F}_{\text {ex }}$, that we will also denote with $\prec$, is defined by cases. If $\varphi, \psi \in \mathcal{F}$, then $\varphi \prec \psi$ iff $(\Rightarrow \varphi) \prec(\Rightarrow \psi)$. If $\varphi \in \mathcal{F}$ and $\psi \notin \mathcal{F}$, then $\varphi \prec \psi$ holds and $\psi \prec \varphi$ does not. If $\varphi, \psi \notin \mathcal{F}$, then $\varphi \prec \psi$ holds if $\operatorname{qf}(\varphi) \prec$ qf $\operatorname{qf}(\psi)$. When $\varphi \prec \psi$, we say that $\varphi$ is of lower rank than $\psi$. Clearly, if the order $\prec$ on sequents is well-founded, then so is the order $\prec$ on $\mathcal{F}_{\text {ex}}$.

\section{Uniform interpolants}

Let $\mathcal{R}$ be a terminating calculus. Recall that $\mathcal{R}_{\text {ins }}$ denotes the set of instances of rules in $\mathcal{R}$. A universal interpolant assignment for $\mathcal{R}$, assigns, for every atom $p$, sequent $S$ in $\mathcal{L}$ and for every $R$ being either $\varnothing$ or an instance of a rule in $\mathcal{R}$ backwards applicable to $S$, to the expression $\forall p R$ a formula in $\mathcal{F}_{\text {ex }}$ that is of lower rank than $\forall p S$. For free sequents $S$ the interpolant assignment assigns formulas in $\mathcal{F}_{\text {ex }}$ of lower rank than $\forall p S$ to expressions $\forall \stackrel{\varnothing}{p} S$.

If $\varphi$ is the formula chosen for $\forall p h$, then we write $\forall p R \sim_{0}^{R} \varphi$. We define an equivalence $\sim$ on formulas in $\mathcal{L}_{\text {ex }}$ that is the smallest equivalence relation that commutes with the connectives and modal operator and furthermore satisfies:

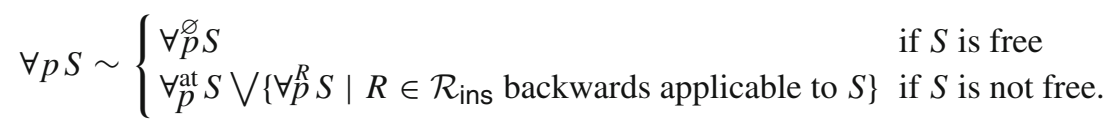

The first disjunct is defined as

$$
\forall_{p}^{\text {at }} S \equiv_{\text {def }} I\left(\left\{q \in S^{a} \mid q \neq p\right\} \Rightarrow\left\{q \in S^{S} \mid q \neq p\right\}\right)
$$


Observe that there could be more than one instance of a single rule $R$ which has $S$ as a conclusion, in which case every instance corresponds to a separate disjunct of the interpolant.

Remark 1 Because of the definition of universal interpolant assignments, for every sequent $S$ minimal in order $\prec$, the formula assigned to $\forall p S$ belongs to $\mathcal{L}$. Therefore one can show by induction along $\prec$ that for every sequent $S$ and atoms $p_{1} \ldots p_{n}$ there exists a formula in $\mathcal{L}$ denoted by $\left\ulcorner\forall p_{1} \ldots p_{n} S\right\urcorner$ such that $\forall p_{1} \ldots p_{n} S \sim\left\ulcorner\forall p_{1} \ldots p_{n} S\right\urcorner$.

Example 1 Suppose the calculus only contains the rule $\mathcal{R}$ for conjunction on the right as given in (2) and there exists an ordering in which sequents with more connectives come after sequents with less connectives. Let $S$ be the sequent $\left(\Rightarrow \varphi_{1} \wedge \psi_{1}, \varphi_{2} \wedge \psi_{2}\right)$ and $R_{i}$ stand for the instance of $\mathcal{R}$ with $\varphi_{i} \wedge \psi_{i}$ as the main formula. By the above definition,

$$
\forall p S \sim \forall p^{R_{1}} S \vee \forall p^{2} S
$$

Let $S_{\varphi_{1}}=\left(\Rightarrow \varphi_{1}, \varphi_{2} \wedge \psi_{2}\right)$ and $S_{\psi_{1}}=\left(\Rightarrow \psi_{1}, \varphi_{2} \wedge \psi_{2}\right)$, and similarly for $S_{\varphi_{2}}$ and $S_{\psi_{2}}$. The standard interpolant assignment introduced below, assigns $\forall p S_{\varphi_{1}} \wedge \forall p S_{\psi_{1}}$ to $\forall p^{1} S$ and $\forall p S_{\varphi_{2}} \wedge \forall p S_{\psi_{2}}$ to $\forall p^{R} S$. This implies that

$$
\forall p S \sim \forall_{p}^{\text {at }} S \vee\left(\forall p S_{\varphi_{1}} \wedge \forall p S_{\psi_{1}}\right) \vee\left(\forall p S_{\varphi_{2}} \wedge \forall p S_{\psi_{2}}\right)
$$

\subsection{The interpolant properties}

A given formula $\varphi$ in $\mathcal{F}$ has uniform interpolants if for every atom $p$ there exist two formulas in $\mathcal{F}$, denoted $\forall p \varphi$ and $\exists p \varphi$, such that for all $\psi$ not containing $p$ :

$$
\vdash \psi \rightarrow \varphi \Leftrightarrow \vdash \psi \rightarrow \forall p \varphi \quad \vdash \varphi \rightarrow \psi \Leftrightarrow \vdash \exists p \varphi \rightarrow \psi .
$$

Given a formula $\varphi$, its universal uniform interpolant with respect to $p_{1} \ldots p_{n}$ is $\forall p_{1} \ldots p_{n}(\Rightarrow \varphi)$, which we write as $\forall p_{1} \ldots p_{n} \varphi$. As we only consider classical logics we can take $\neg \forall \neg$ for $\exists$ and the above is equivalent to

$$
\vdash \forall p \varphi \rightarrow \varphi \quad \vdash \psi \rightarrow \varphi \Rightarrow \vdash \psi \rightarrow \forall p \varphi
$$

Because in this paper we express most results in terms of universal interpolants, we usually omit the word universal and just speak of uniform interpolants.

A logic has uniform interpolation if all its formulas have uniform interpolants. This is equivalent to the statement that for every atom $p$ and for any set of atoms $\mathcal{P}$ not containing $p$, the embedding of $\mathcal{F}(\mathcal{P})$ into $\mathcal{F}(\mathcal{P} \cup\{p\})$ has a right and a left adjoint.

As will be shown in Lemma 1, in the approach via sequents, $(\forall)$ is replaced by the following requirements, the interpolant properties.

( $\forall \mathrm{l})$ for all $p: \vdash S^{a}, \forall p S \Rightarrow S^{s}$;

$(\forall \mathrm{r})$ for all $p: \vdash S^{r} \cdot\left(\Rightarrow \forall p S^{i}\right)$ if $S$ is derivable and $S^{r}$ does not contain $p$. 
A calculus $\mathrm{G}$ has uniform interpolation if $(\forall \mathrm{l})$ and $(\forall \mathrm{r})$ hold, where $\vdash$ denotes $\vdash_{\mathrm{G}}$.

Property $(\forall \mathrm{l})$ is the independent (from partitions) interpolant property, and $(\forall \mathrm{r})$ is the dependent interpolant property. For every such property and any $R \in \mathcal{R}_{\text {ins }} \cup\{\varnothing\}$, the $R$-variant is the result of replacing quantifier $\forall p$ in the property by $\forall p$. A partition $\left(S^{r}, S^{i}\right)$ of $S$ satisfies the interpolant properties if, in the case of the independent property, $S$ satisfies it (in which case we also say that $S$ satisfies it), and in case of the dependent property, it holds for that particular partition. A sequent satisfies a property if every possible partition of the sequent satisfies it.

Remark 2 Observe that the independent interpolant property holds for a sequent $S$ if and only if the $R$-variant of the independent interpolant property holds for all $R$ backwards applicable to $S$ and the independent interpolant property in which $\forall p$ is replaced by $\forall_{p}^{\text {at }}$ holds. If the $R$-variant of the dependent interpolant property holds for partition $\left(S^{r}, S^{i}\right)$ for at least one $R$ that is backwards applicable to $S^{i}$, then the property holds for $\left(S^{r}, S^{i}\right)$.

Lemma 1 If all sequents satisfy the interpolant properties, then $\mathrm{L}$ has uniform interpolation.

Proof We have to show that $(\forall)$ holds. Consider $S=(\Rightarrow \varphi)$. Hence $\forall p \varphi=\forall p S$ by definition. By $(\forall 1), \vdash I(\forall p \varphi \Rightarrow \varphi)$, that is, $\forall p \varphi \rightarrow \varphi$ is derivable.

Consider a $\psi$ not containing $p$ such that $\vdash \psi \rightarrow \varphi$. Let $S=(\psi \Rightarrow \varphi)$ and consider the partition $\left(S^{r}, S^{i}\right)$, where $S^{r}=(\psi \Rightarrow)$ and $S^{i}=(\Rightarrow \varphi)$. Hence $\forall p \varphi=\forall p S^{i}$ by definition. And $\vdash(\psi \Rightarrow) \cdot(\Rightarrow \forall p \varphi)$ by $(\forall \mathrm{r})$. Therefore $(\psi \rightarrow \forall p \varphi)$ is derivable.

A fact that we will often be used is that all free sequents satisfy the dependent interpolant properties.

\subsection{Soundness}

In order to divide the proof of the main theorems in separate parts we introduce the following three properties of rules $\mathcal{R}$.

$(\mathrm{IP})_{\mathcal{R}} \quad\left\{S_{j} \cdot\left(\forall p S_{j} \Rightarrow\right) \mid 1 \leq j \leq n\right\} \vdash S_{0} \cdot\left(\forall \stackrel{R}{p} S_{0} \Rightarrow\right)$ for every instance $R=S_{1} \ldots S_{n} / S_{0}$ of $\mathcal{R}$.

$(\mathrm{DPB})_{\mathcal{R}}$ For every instance $S_{1} \ldots S_{n} / S_{0}$ of $\mathcal{R}$ and every $p$-partition $\left(S_{0}^{r}, S_{0}^{i}\right)$ of $S_{0}$ such that $\mathcal{R}$ is backwards applicable to $S_{0}^{i}$, there exists a partition of $\mathcal{R}$ such that $\left\{S_{j}^{r} \cdot\left(\Rightarrow \forall p S_{j}^{i}\right) \mid 1 \leq j \leq n\right\} \vdash S_{0}^{r} \cdot\left(\Rightarrow \forall p S_{0}^{i}\right)$, where $R$ is an instance of $\mathcal{R}$ with conclusion $S_{0}^{i}$.

$(\mathrm{DPN})_{\mathcal{R}}$ For every instance $S_{1} \ldots S_{n} / S_{0}$ of $\mathcal{R}$ and every $p$-partition $\left(S_{0}^{r}, S_{0}^{i}\right)$ of $S_{0}$ such that $S_{0}^{i}$ is not empty and $\mathcal{R}$ is not backwards applicable to $S_{0}^{i}$, if all sequents lower than $S_{0}$ satisfy the interpolant properties, then the sequent $S_{0}^{r} \cdot\left(\Rightarrow \forall p S_{0}^{i}\right)$ is derivable.

((IP) stands for independent property, (DP) for dependent property, B for backwards.)

(FP) If $S$ is free and all sequents lower than $S$ satisfy the interpolant properties, then $S$ satisfies the independent interpolant property. 
An interpolant assignment is sound for a given calculus if (FP) holds and (IP) $)_{\mathcal{R}}$, $(\mathrm{DPB})_{\mathcal{R}}$ and $(\mathrm{DPN})_{\mathcal{R}}$ hold for every rule $\mathcal{R}$ in the calculus.

Since free sequents trivially satisfy the dependent interpolant property, this seems to suggest that one could always take $\perp$ for the interpolant assignment of free sequents. Free sequents do, however, also play a role in the dependent interpolant property, because the partition of a sequent might be such that $S^{i}$ is a free sequent. Therefore this simple interpolant assignment for free sequents might not always be possible.

Lemma 2 If a logic $\mathrm{L}$ has a terminating calculus that is closed under weakening and for which there exists a sound interpolant assignment, then all sequents satisfy the interpolant properties.

Proof We have to show that under the assumptions in the lemma, the interpolant properties hold for any $p$-partition $\left(S^{r}, S^{i}\right)$ of any sequent $S$. Free sequents satisfy the dependent property in a trivial way, as they are not derivable. Therefore, using Remark 2, it suffices to show that for any sequent $S$ and any $p$-partition $\left(S^{r}, S^{i}\right)$ of $S$ :

1. $\vdash S^{a}, \forall_{p}^{\text {at }} S \Rightarrow S^{s}$ if $S$ is not free,

2. $\vdash S^{a}, \forall p h S S^{s}$ for all $R$ backwards applicable to $S$, if $S$ is not free,

3. $\vdash S^{r} \cdot\left(\Rightarrow \forall p S^{i}\right)$ in case $S$ is derivable,

4. $S$ satisfies the independent interpolant property if $S$ is free.

For 1 , note that in case $\forall_{p}^{\text {at }} S$ is empty, 1 clearly holds. Therefore assume $\forall_{p}^{\text {at }} S$ is not empty, say it is of the form $\bigwedge q_{i} \rightarrow \bigvee r_{j}$, where the disjunct or conjunct may be empty, but not both. Then $S^{a}, \forall_{p}^{\text {at }} S \Rightarrow S^{s}$ is clearly derivable.

Statements 2 and 3 are proved by induction on the well-founded order $\prec$ on $\mathcal{F}_{\text {ex }}$ defined in Sect. 2.5. Recall that in every rule of the calculus the premisses come before the conclusion in this order, and all proper subsequents of a sequent come before that sequent.

Sequents $S$ that are not free and minimal in the order have to be axioms. Thus 2 follows from $(\mathrm{IP})_{\mathcal{R}}$ as the set corresponding to the premisses is empty since an axiom has no premisses. For 3 , since $S$ is minimal in the order, $S^{i}=S$ or $S^{i}$ is the empty sequent. In the first case, for the same reason as in the case of $(\mathrm{IP})_{\mathcal{R}}$, property $(\mathrm{DPB})_{\mathcal{R}}$ implies that $\vdash S^{r} \cdot\left(\Rightarrow \forall p S^{i}\right)$. In the last case, because $S^{r}=S$ and $S$ is derivable, also the weakening $S^{r} \cdot\left(\Rightarrow \forall p S^{i}\right)$ is derivable. Hence 3 holds.

For the induction step, assume that all sequents lower than $S$ satisfy the interpolant properties. For 2, consider an instance $R=\left(S_{1} \ldots S_{n} / S\right)$ of a rule. The induction hypothesis implies that the interpolant properties hold for $S_{1}, \ldots, S_{n}$. Therefore 2 follows from $(\mathrm{IP})_{\mathcal{R}}$. For 3 , assume that $S$ is derivable and let $\mathcal{R}$ be a rule or axiom that is backwards applicable to $S$, and consider a partition $\left(S^{r}, S^{i}\right)$ of $S$ and a corresponding partition of $\mathcal{R}$. In case $S^{i}$ is the empty sequent, $S^{r}=S$ and 3 clearly holds. Thus assume $S^{i}$ is not empty. In case $\mathcal{R}$ is backwards applicable to $S^{i}$, then 3 follows from (DPB $)_{\mathcal{R}}$. In case $\mathcal{R}$ is not backwards applicable to $S^{i},(\mathrm{DPN})_{\mathcal{R}}$ applies.

Finally, 4 is proved by induction to $\prec$. If $S$ is minimal in the ordering, then it satisfies the independent interpolant property by (FP). In the induction step we assume that all sequents lower than $S$ satisfy the interpolant properties. Hence we can apply (FP) again to show that the independent interpolant property holds for $S$. 
Theorem 1 If a logic $\mathrm{L}$ has a terminating calculus that is closed under weakening and for which there exists a sound interpolant assignment, then $\mathrm{L}$ has uniform interpolation.

Proof This follows from Lemmas 1 and 2.

\section{Standard assignments for propositional logic}

In this section we define a specific universal interpolant assignment for propositional logic inspired by Pitts' method. It is proved to be sound for focussed axioms and rules.

For an instance

$$
\frac{S_{1} \quad S_{2} \quad \ldots \quad S_{n}}{S_{0}} R
$$

of a $n$-premiss rule the standard universal interpolant assignment is

$$
\forall p S_{0} \sim_{0} \forall p S_{1} \wedge \forall p S_{2} \wedge \cdots \wedge \forall p S_{n}
$$

If all instances of a schematic rule $\mathcal{R}$ have a standard interpolant assignment, then the interpolant assignment for $\mathcal{R}$ is standard.

For instances $R$ of focussed axioms consisting of sequent $S$, the standard interpolant assignment is defined as

$$
\forall p R \sim_{0} \top
$$

For free sequents $S$ the standard interpolant assignment is

$$
\forall \stackrel{\varnothing}{p} S \equiv_{\text {def }}\left(\left\{\varphi \in S^{a} \mid \varphi \text { does not contain } p\right\} \Rightarrow\left\{\psi \in S^{S} \mid \psi \text { does not contain } p\right\}\right) .
$$

We fix a partitioning of instances $R$ of focussed rules $\mathcal{R}$ with the property that if $\mathcal{R}$ is backwards applicable to $\left(S \cdot S_{0}\right)^{i}$, then $R^{i}$ is an instance of $\mathcal{R}$ and if $\mathcal{R}$ is not backwards applicable to $\left(S \cdot S_{0}\right)^{i}$, then $R^{r}$ is an instance of $\mathcal{R}$. Therefore we define a partition $\operatorname{pt}(R)$

$$
\frac{\left(\left(S \cdot S_{1}\right)^{r},\left(S \cdot S_{1}\right)^{i}\right) \ldots\left(\left(S \cdot S_{n}\right)^{r},\left(S \cdot S_{n}\right)^{i}\right)}{\left(\left(S \cdot S_{0}\right)^{r},\left(S \cdot S_{0}\right)^{i}\right)} \operatorname{pt}(R)
$$

of an instance $\left(S \cdot S_{1}\right), \ldots,\left(S \cdot S_{n}\right) /\left(S \cdot S_{0}\right)$ of a focussed rule to be standard if for all $1 \leq j \leq n$ :

$$
\left(S \cdot S_{j}\right)^{i}= \begin{cases}\left(S^{i} \cdot S_{j}\right) & \text { if }\left(S \cdot S_{0}\right)^{i}=S^{i} \cdot S_{0} \text { for some } S^{i} \subseteq S \\ \left(S \cdot S_{0}\right)^{i} & \text { otherwise. }\end{cases}
$$

$\left(S \cdot S_{j}\right)^{r}$ is defined to be the unique sequent such that $\left(\left(S \cdot S_{j}\right)^{r},\left(S \cdot S_{j}\right)^{i}\right)$ is a partition of $\left(S \cdot S_{j}\right)$. If the first case in the definition of $\left(S \cdot S_{j}\right)^{i}$ does not apply, then as $S_{0}$ contains exactly one formula, $S_{0} \subseteq\left(S \cdot S_{0}\right)^{r}$ and $\left(S \cdot S_{0}\right)^{i} \subseteq S$. Therefore 


$$
\left(S \cdot S_{j}\right)^{r}= \begin{cases}\left(S \cdot S_{0}\right)^{r} & \text { if }\left(S \cdot S_{0}\right)^{i}=S^{i} \cdot S_{0} \text { for some } S^{i} \subseteq S \\ \left(S^{r} \cdot S_{j}\right) & \text { if }\left(S \cdot S_{0}\right)^{r}=S^{r} \cdot S_{0} \text { for some } S^{r} \subseteq S .\end{cases}
$$

Example 2 Consider the following instance $R$ of the rule $\mathcal{R}$ for conjunction on the right:

$$
\frac{\Gamma \Rightarrow \varphi_{1}, \Delta \Gamma \Rightarrow \varphi_{2}, \Delta}{\Gamma \Rightarrow \varphi_{1} \wedge \varphi_{2}, \Delta}
$$

Thus $S_{0}=\left(\Rightarrow \varphi_{1} \wedge \varphi_{2}\right), S_{j}=\left(\Rightarrow \varphi_{j}\right)$ for $j=1,2$ and $S=(\Gamma \Rightarrow \Delta)$. We will describe two standard partitions of the rule, one corresponding to the first case in the definition above and the other to the second. First, consider partition $\left(S_{0}^{r}, S_{0}^{i}\right)$ of the conclusion $S_{0}$, where $\left(S \cdot S_{j}\right)^{i}=\left(\Rightarrow \varphi_{1} \wedge \varphi_{2}\right)$. From $S_{0} \subseteq\left(S \cdot S_{j}\right)^{i}$ it follows that $\left(S \cdot S_{j}\right)^{i}=\left(\Rightarrow \varphi_{j}\right)$ for $j=1,2$ and $S_{j}^{r}=S$ for $j=0,1,2$.

Second, assume $\Delta \neq \varnothing$ and the formula in $S_{0}$ does not occur in $\Delta$, and consider partition $\left(S_{0}^{r}, S_{0}^{i}\right)$ of $S_{0}$, where $\left(S \cdot S_{0}\right)^{i}=(\Rightarrow \Delta)$. Thus $\left(S \cdot S_{0}\right)^{r}=\left((\Gamma \Rightarrow) \cdot S_{0}\right)$. As $S_{0} \nsubseteq\left(S \cdot S_{0}\right)^{i},\left(S \cdot S_{j}\right)^{i}=S^{i}$ for $j=0,1,2,\left(S \cdot S_{0}\right)^{r}=\left(\Gamma \Rightarrow \varphi_{1} \wedge \varphi_{2}\right)$, and $\left(S \cdot S_{j}\right)^{r}=\left(\Gamma \Rightarrow \varphi_{j}\right)$ for $j=1,2$.

Observe that in the first case $R^{i}$ is an instance of $\mathcal{R}$, and in the second case $R^{r}$ is. Generalising this gives the following lemma.

Lemma 3 For any instance $R=\left(\left(S \cdot S_{1}\right) \ldots\left(S \cdot S_{n}\right) /\left(S \cdot S_{0}\right)\right)$ of a focussed rule $\mathcal{R}$ with a standard partition, either $S_{0} \subseteq\left(S \cdot S_{0}\right)^{i}$ and $R^{i}$ is an instance of $\mathcal{R}$ or $S_{0} \subseteq\left(S \cdot S_{0}\right)^{r}$ and $R^{r}$ is an instance of $\mathcal{R}$.

\subsection{Properties of focussed rules}

Lemma 4 For any instance $S_{1} \ldots S_{n} / S_{0}$ of a focussed rule and any formulas $\varphi_{1}, \ldots, \varphi_{n}$ :

$$
\left\{S_{j} \cdot\left(\varphi_{j} \Rightarrow\right) \mid j=1, \ldots, n\right\} \vdash_{\mathrm{CPC}}^{\mathcal{R}} S_{0} \cdot\left(\bigwedge_{j=1}^{n} \varphi_{j} \Rightarrow\right)
$$

Proof Clearly, $\left\{S_{1}, \ldots, S_{n}\right\} \vdash_{\text {CPC }}^{\mathcal{R}} S_{0}$. Let $S=\left(\bigwedge_{j=1}^{n} \varphi_{j} \Rightarrow\right)$. Since $\mathcal{R}$ is focussed, we have $\left\{S \cdot S_{1}, \ldots, S \cdot S_{n}\right\} \vdash_{\mathrm{CPC}}^{\mathcal{R}} S \cdot S_{0}$. Since $S_{j} \cdot\left(\varphi_{j} \Rightarrow\right) \vdash_{\mathrm{CPC}}^{\mathcal{R}} S_{j} \cdot S$, the desired follows.

Lemma 5 For all formulas $\varphi_{1}, \ldots, \varphi_{n}$ and any partition $\left(S^{r}, S^{i}\right)$ of the conclusion of an instance $S_{1} \ldots S_{n} / S$ of a focussed rule $\mathcal{R}$ which is backwards applicable to $S^{i}$ :

$$
\left\{S_{j}^{r} \cdot\left(\Rightarrow \varphi_{j}\right) \mid j=1, \ldots, n\right\} \vdash_{\mathrm{CPC}}^{\mathcal{R}} S^{r} \cdot\left(\Rightarrow \bigwedge_{j=1}^{n} \varphi_{j}\right) .
$$


Proof Consider the standard partition of $\mathcal{R}$. As $\mathcal{R}$ is backwards applicable to $S^{i}$, $S^{r}=S_{j}^{r}$, which immediately implies what we had to show.

Lemma 6 For all sequents $S$ and any standard partition of an instance $R=$ $S_{1} \ldots S_{n} / S_{0}$ of a focussed rule $\mathcal{R}$ which is not backwards applicable to $S_{0}^{i}$ :

$$
\left\{S_{j}^{r} \cdot S \mid j=1, \ldots, n\right\} \vdash_{\mathrm{CPC}}^{\mathcal{R}} S_{0}^{r} \cdot S .
$$

Proof As $\mathcal{R}$ is not backwards applicable to $S_{0}^{i}, R^{r}$ is an instance of $\mathcal{R}$ by Lemma 3, using the standard partition of $\mathcal{R}$. Because the rule $\mathcal{R}$ is focussed, $\left\{S \cdot S_{1}^{r}, \ldots, S \cdot S_{n}^{r}\right\} / S \cdot S_{0}^{r}$ is an instance of $\mathcal{R}$, which is what we had to show.

Lemma 7 For any focussed rule $\mathcal{R}$ in any calculus for a logic, $(I P)_{\mathcal{R}}$ holds.

Proof Let $R=S_{1} \ldots S_{n} / S_{0}$ be an instance of $\mathcal{R}$. Then $\forall p S_{0} \sim \bigwedge_{j=1}^{n} \forall p S_{j}$. Thus by letting $\varphi_{j}=\forall p S_{j}$, Lemma 4 and the fact that $\vdash_{\mathrm{CPC}}^{\mathcal{R}} \subseteq \vdash$ can be applied to obtain $($ IP) $\mathcal{R}$.

Lemma 8 For any focussed rule $\mathcal{R}$ in any terminating calculus with a standard interpolant assignment, $(D P B)_{\mathcal{R}}$ holds.

Proof Consider an instance $R=S_{1} \ldots S_{n} / S$ of $\mathcal{R}$ and let $\left(S^{r}, S^{i}\right)$ be a partition of $S$ such that $\mathcal{R}$ is backwards applicable to $S^{i}$. If $\mathcal{R}$ is an axiom, then $\forall p R \sim \top$ and thus $\forall p S \sim \top$. Similarly for $\forall p S^{i}$. Therefore (DPB) $)_{\mathcal{R}}$ clearly holds for sequents that are instances of axioms. Suppose $\mathcal{R}$ is not an axiom. By Lemma 3 for the standard partition of $\mathcal{R}$ there is an instance $R$ of $\mathcal{R}$ with conclusion $S^{i}$ and premisses $S_{1}^{i}, \ldots, S_{n}^{i}$. Therefore $\forall p S^{i} \sim \bigwedge_{j=1}^{n} \forall p S_{j}^{i}$. Thus by letting $\varphi_{j}=\forall p S_{j}^{i}$, Lemma 5 can be applied to obtain $(\mathrm{DPB})_{\mathcal{R}}$.

Lemma 9 For every instance $S_{1} \ldots S_{n} / S_{0}$ of a focussed rule $\mathcal{R}$ in any terminating calculus with a standard interpolant assignment and every p-partition $\left(S_{0}^{r}, S_{0}^{i}\right)$ of $S_{0}$ such that $S_{0}^{i}$ is not empty and $\mathcal{R}$ is not backwards applicable to $S_{0}^{i}$ :

$$
\left\{S_{j}^{r} \cdot\left(\Rightarrow \forall p S_{j}^{i}\right) \mid 1 \leq j \leq n\right\} \vdash_{\mathrm{CPC}}^{\mathcal{R}} S_{0}^{r} \cdot\left(\Rightarrow \forall p S_{0}^{i}\right) .
$$

Proof First consider the case that $\mathcal{R}$ is an axiom. If it is of the form $(\Gamma, \perp \Rightarrow \Delta)$ or $(\Gamma \Rightarrow \top, \Delta)$, then the fact that $S_{0}^{i}$ is not instance of it implies that $S_{0}^{r} \cdot\left(\Rightarrow \forall p S_{0}^{i}\right)$ is an instance of the axiom, and we are done. If it is of the form $(\Gamma, q \Rightarrow q, \Delta)$, then $q \neq p$. For if $q=p$, as $S_{0}^{r}$ does not contain $p,(q \Rightarrow q)$ would be a subsequent of $S_{0}^{i}$, quod non. If $q \Rightarrow q$ is a subsequent of $S_{0}^{r}$, then $S_{0}^{r} \cdot\left(\Rightarrow \forall p S_{0}^{i}\right)$ is an instance of the axiom, and we are done again. Therefore consider the case that $q \Rightarrow q$ is neither a subsequent of $S_{0}^{i}$ nor of $S_{0}^{r}$. If $S_{0}^{i}$ is free, the standard interpolant assignment implies that $\vdash_{\mathrm{CPC}}^{\mathcal{R}} S_{0}^{r} \cdot\left(\Rightarrow \forall p S_{0}^{i}\right)$. If $S_{0}^{i}$ is not free, it suffices to prove that $\vdash_{\mathrm{CPC}}^{\mathcal{R}} S_{0}^{r} \cdot\left(\Rightarrow \forall_{p}^{\text {at }} S_{0}^{i}\right)$, which follows from the fact that $S_{0}^{r} \cdot\left(\Rightarrow \forall_{p}^{\text {at }} S_{0}^{i}\right)$ contains the sequent $q \Rightarrow q$.

The case that $\mathcal{R}$ is not an axiom remains. By Lemma 6 , the fact that $\mathcal{R}$ is not backwards applicable to $S_{0}^{i}$ implies that for any standard partition and any sequent $S:\left\{S \cdot S_{j}^{r} \mid j=1, \ldots, n\right\} \vdash_{\mathrm{CPC}}^{\mathcal{R}} S \cdot S_{0}^{r}$. Lemma 3 implies that $S_{0}^{i}=S_{j}^{i}$, and thus $\left(\Rightarrow \forall p S_{j}^{i}\right)=\left(\Rightarrow \forall p S_{0}^{i}\right)$ for all $j=1, \ldots, n$, from which the lemma follows. 
Lemma 10 For any focussed rule $\mathcal{R}$ in any terminating calculus with a standard interpolant assignment, $(D P N)_{\mathcal{R}}$ holds.

Proof By Lemma 9.

Lemma 11 In any terminating calculus with a standard interpolant assignment (FP) holds.

Proof Let $S$ be a free sequent. We have to show that $S$ satisfies the independent interpolant property, that is, that $S^{a}, \forall p S \Rightarrow S^{S}$ is derivable. By definition $\forall p S=$ $\forall \stackrel{\varnothing}{p} S$, where

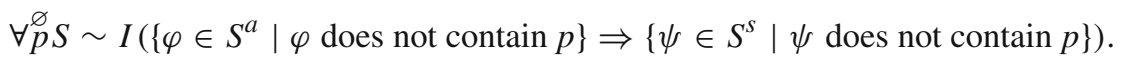

Therefore $S^{a}, \forall p S \Rightarrow S^{s}$ is equivalent to a sequent $S^{a},(\bigwedge \Gamma \rightarrow \bigvee \Delta) \Rightarrow S^{s}$ for some $\Gamma \subseteq S^{a}$ and $\Delta \subseteq S^{s}$, which is clearly derivable, even if $\Gamma$ or $\Delta$ is empty.

Theorem 2 A logic $\mathrm{L}$ with a terminating calculus in which all rules and axioms are focussed has uniform interpolation.

Proof First observe that such a calculus is closed under weakening. By Theorem 1 it suffices to prove that the interpolant assignment is sound. This follows from Lem$\operatorname{mas} 7,8,10$, and 11 .

\subsection{Classical logic}

To establish that classical propositional logic has uniform interpolation is not hard. More generally, it is known that if a logic has interpolation and is locally tabular ${ }^{1}$, properties that indeed hold for CPC, then it has uniform interpolation. However, with the methods developed in the previous sections one can also easily infer that CPC has uniform interpolation from the existence of a terminating calculus for the logic in which all axioms and rules are focussed. The propositional part, G3p, of the calculus G3 from [8] which is given in Fig. 1 has these properties. It is terminating (using the natural ordering given by the number of symbols in a sequent) and the axioms and rules are clearly focussed. The calculus has no structural rules, but they are admissible in it, as is the cut rule.

Theorem 3 Classical propositional logic has uniform interpolation.

Proof By Theorem 2 and the fact that the terminating calculus G3p is sound and complete for CPC.

Note that for the above result one cannot use the propositional part of Gentzen's LK or other calculi that contain the Cut Rule, as such calculi may not be terminating.

\footnotetext{
${ }^{1}$ For any finite set of variables there are only finitely many nonequivalent formulas in those variables.
} 


$$
\begin{array}{ll}
\Gamma, p \Rightarrow p, \Delta \quad \text { At }(\Gamma \cup \Delta \text { atoms }) & \Gamma, \perp \Rightarrow \Delta \quad L \perp \\
\frac{\Gamma \Rightarrow \varphi \quad \Gamma \Rightarrow \psi, \Delta}{\Gamma \Rightarrow \varphi \wedge \psi, \Delta} \mathrm{R} \wedge & \frac{\Gamma, \varphi, \psi \Rightarrow \Delta}{\Gamma, \varphi \wedge \psi \Rightarrow \Delta} \mathrm{L} \wedge \\
\frac{\Gamma \Rightarrow \varphi, \psi, \Delta}{\Gamma \Rightarrow \varphi \vee \psi, \Delta} \mathrm{R} \vee & \frac{\Gamma, \varphi \Rightarrow \Delta \quad \Gamma, \psi \Rightarrow \Delta}{\Gamma, \varphi \vee \psi \Rightarrow \Delta} \mathrm{L} \vee \\
\frac{\Gamma, \varphi \Rightarrow \psi, \Delta}{\Gamma \Rightarrow \varphi \rightarrow \psi, \Delta} \mathrm{R} \rightarrow & \frac{\Gamma \Rightarrow \varphi, \Delta \quad \Gamma, \psi \Rightarrow \Delta}{\Gamma, \varphi \rightarrow \psi \Rightarrow \Delta} \mathrm{L} \rightarrow \\
\frac{\Gamma, \varphi \Rightarrow \Delta}{\Gamma \Rightarrow \neg \varphi, \Delta} \mathrm{R} \neg & \frac{\Gamma \Rightarrow \varphi, \Delta}{\Gamma, \neg \varphi \Rightarrow \Delta} \mathrm{L} \neg
\end{array}
$$

Fig. 1 The Gentzen calculus G3p

\section{Standard assignment for modal logics}

In this section we extend the method developed above to modal logic by extending the class of rules to which Theorem 2 applies. We use the convention that $\square S=$ $\left(\left\{\square \varphi \mid \varphi \in S^{a}\right\} \Rightarrow\left\{\square \psi \mid \psi \in S^{s}\right\}\right)$, implying that $\square(\Gamma \Rightarrow)=(\square \Gamma \Rightarrow)$ and $\square(\Rightarrow \Delta)=(\Rightarrow \square \Delta)$.

A focussed rule is defined as before, in Sect. 2.2, except that it is required that the formula in $S_{0}$ is not boxed. A rule $\mathcal{R}$ is a focussed modal rule if it is of the form

$$
\frac{\square S_{1} \cdot S_{0}}{S_{2} \cdot \square S_{1} \cdot \square S_{0}} \mathcal{R}
$$

for certain sequents $S_{0}, S_{1}, S_{2}$ such that

- $S_{2}^{a}, S_{2}^{s}$ both consist of a meta-multiset;

- $S_{1}^{a}, S_{1}^{s}$ both consist of at most one element, which is a meta-multiset;

- $S_{0}^{a}, S_{0}^{s}$ both consist of meta-multisets and meta-atoms;

- $S_{0}$ contains exactly one meta-atom;

○ no meta-multiset occurs in $S_{2} \cdot S_{1} \cdot S_{0}$ more than once;

○ if $S_{i}^{a}$ contains a meta-multiset for $i=0$ or $i=1$, then the meta-multiset in $S_{2}^{a}$ ranges over multisets of atoms;

○ if $S_{i}^{S}$ contains a meta-multiset for $i=0$ or $i=1$, then the meta-multiset in $S_{2}^{S}$ ranges over multisets of atoms.

Thus $S_{1}$ may be empty, but $S_{2}$ and $S_{0}$ may not. Note that $S_{2}$ is of the form $\Lambda \Rightarrow$ $\Theta$ for two meta-multiset symbols $\Lambda$ and $\Theta$ that do not occur in $S_{1}$ or $S_{0}$. Given a focussed modal rule $\mathcal{R}$, let $\mathcal{R}^{+}$denote the rule obtained from $\mathcal{R}$ by omitting the last two restrictions about the multisets in $S_{2}$. In Lemma 21 it is shown that $\mathcal{R}^{+}$is not stronger than $\mathcal{R}$. The reason to work with the restricted rules is to simplify the proofs in this section.

Up to the renaming of meta-symbols there are eight possibilities for $S_{0}$ and four for $S_{1}$. To each possibility corresponds a name, which is a set consisting of the elements $\mathrm{L}, \mathrm{I}, \mathrm{R}, \mathrm{r}, \mathrm{W}, \mathrm{E}$, the lower case letters referring to the position of the atom in $S_{0}$ and the 
upper case letters to the presence and position of the multisets in $S_{0}$ (left and right) and in $S_{1}$ (west and east). For brevity we denote the sets as strings, so that, for example, Lr should be read as $\{L, r\}$.

$\begin{array}{llllll}\text { name } & S_{0} & \text { name } & S_{0} & \text { name } & S_{1} \\ \text { LIR } & \Gamma, p \Rightarrow \Delta & \text { LrR } & \Gamma \Rightarrow p, \Delta & \varnothing & (\Rightarrow) \\ \text { LI } & \Gamma, p \Rightarrow & \text { Lr } & \Gamma \Rightarrow p & \text { W } & (\Pi \Rightarrow) \\ \text { IR } & p \Rightarrow \Delta & \text { rR } & \Rightarrow p, \Delta & \mathrm{E} & (\Rightarrow \Sigma) \\ \text { I } & p \Rightarrow & \mathrm{r} & \Rightarrow p & \text { WE } & (\Pi \Rightarrow \Sigma)\end{array}$

The terminology for the 32 focussed modal rules is as follows. Consider a focussed modal rule as in (3) and let $\mathrm{x}$ and $\mathrm{y}$ be the names in the list above for $S_{0}$ and $S_{1}$, respectively. Then the name of the rule with this $S_{0}$ and $S_{1}$ is $\mathcal{R}_{\mathrm{x} \cup \mathrm{y}}$, where $\mathrm{x} \cup \mathrm{y}$ is again abbreviated as a string. For example, $\mathcal{R}_{\mathrm{WIR}}$ and $\mathcal{R}_{\mathrm{Lr}}$ denote the following rules, where the latter is the well-known rule for $\mathrm{K}$.

$$
\frac{\square \Pi, p \Rightarrow \Delta}{\Lambda, \square \Pi, \square p \Rightarrow \square \Delta, \Theta} \mathcal{R}_{\mathrm{WIR}} \quad \frac{\Gamma \Rightarrow p}{\Lambda, \square \Gamma \Rightarrow \square p, \Theta} \mathcal{R}_{\mathrm{Lr}}
$$

$\mathcal{R}_{\mathrm{Lr}}$ is also denoted by $\mathcal{R}_{\mathrm{K}}$, and a calculus containing that rule is called normal. For subsets $\mathrm{X}, \mathrm{y} \subseteq\{\mathrm{W}, \mathrm{L}, \mathrm{I}, \mathrm{r}, \mathrm{R}, \mathrm{E}\}, \mathcal{R}_{\mathrm{x}}$ is a y-rule if all elements of y occur in $\mathrm{X}$.

The following eight rules are called bad rules: $\mathcal{R}_{\mathrm{WIRx}}$ and $\mathcal{R}_{\mathrm{LrEx}}$, where $\mathrm{X}$ ranges over subsets of $\{W, L, I, r, R, E\}$. Note that $\mathcal{R}_{K}$ is not a bad rule, and neither is any rule that does not contain $\mathrm{W}$ or $\mathrm{E}$.

As for focussed rules we fix a partition of instances of focussed modal rules, for which we have to address a small technical detail first. Given the conclusion of a focussed modal rule $S=\left(S_{2} \cdot \square S_{1} \cdot \square S_{0}\right)$, we assume that for every formula in $S$ it is indicated, although we will not explicitly do so, whether it belongs to $S_{0}, S_{1}$ or $S_{2}$. In this way, for any partition $\left(S^{i}, S^{r}\right)$ of $S, j=0,1,2$ and $x \in\{i, r\}$, there exist unique sequents $S_{j}^{x} \subseteq S_{j}$ ( $\subseteq$ defined in Sect. 2) such that $S^{x}=S_{2}^{x} \cdot \square S_{1}^{x} \cdot \square S_{0}^{x}$. Given this assumption, a partition

$$
\frac{\left(\left(\square S_{1} \cdot S_{0}\right)^{r},\left(\square S_{1} \cdot S_{0}\right)^{i}\right)}{\left(\left(S_{2} \cdot \square S_{1} \cdot \square S_{0}\right)^{r},\left(S_{2} \cdot \square S_{1} \cdot \square S_{0}\right)^{i}\right)} \operatorname{pt}(R)
$$

of an instance $R=\left(\square S_{1} \cdot S_{0} / S_{2} \cdot \square S_{1} \cdot \square S_{0}\right)$ of a focussed modal rule is standard if for $x \in\{i, r\}$ :

$$
\left(\square S_{1} \cdot S_{0}\right)^{x}=\square S_{1}^{x} \cdot S_{0}^{x}
$$

Lemma 12 For any instance $R$ of any focussed modal rule $\mathcal{R}$ with a standard partition, either $R^{i}$ or $R^{r}$ (or both) is an instance of $\mathcal{R}$.

Proof In case $\mathcal{R}$ is an r-rule, either $S_{0}^{i s}$ or $S_{0}^{r s}$ is not empty. In the first case, $R^{i}$ is an instance of $\mathcal{R}$ and in the second case $R^{r}$ is. The case of an I-rule is similar. 
Remark 3 The fact that in focussed modal rules (3) the sequent $S_{2}$ is of the form $\left(\Gamma \Rightarrow \Delta\right.$ ) for two meta-multisets $\Gamma$ and $\Delta$, implies that if $S / S^{\prime}$ is an instance of a focussed modal rule, then so is $S / S^{\prime} \cdot S^{\prime \prime}$ for any sequent $S^{\prime \prime}$.

Lemma 13 If $R=\left(S_{u} / S\right)$ is an instance of a focussed modal rule $\mathcal{R}$ that is not bad, $\left(S^{r}, S^{i}\right)$ is a partition of $S$ such that $\mathcal{R}$ is backwards applicable to $S^{i}$ and $S_{u}^{i}$ is the premiss of $R^{i}$, then one of the following cases hold:

1. $S_{u}^{i} / S^{i}$ is an instance of $\mathcal{R}$,

2. $S_{u}^{i}$ consists of boxed formulas only,

3. $S_{u}^{i}$ has an empty antecedent and $\mathcal{R}$ is an $\mathrm{I}$-rule,

4. $S_{u}^{i}$ has an empty succedent and $\mathcal{R}$ is an $\mathrm{r}$-rule.

Moreover, in cases (2)-(4), for any $\varphi: S_{u}^{i} \cdot(\varphi \Rightarrow) / S^{i} \cdot(\square \varphi \Rightarrow)$ is an instance of $\mathcal{R}$ if $\mathcal{R}$ is an l-rule and $S_{u}^{i} \cdot(\Rightarrow \varphi) / S^{i} \cdot(\Rightarrow \square \varphi)$ is an instance of $\mathcal{R}$ if $\mathcal{R}$ is an r-rule.

Proof We treat the case that $\mathcal{R}$ is an I-rule, the other case is analogous. Thus $\mathcal{R}$ is of the following form, where any of the $\Pi, \Gamma, \Delta, \Sigma$ may be empty.

$$
\frac{\square \Pi, \Gamma, \varphi \Rightarrow \Delta, \square \Sigma}{\Lambda, \square \Pi, \square \Gamma, \square \varphi \Rightarrow \square \Delta, \square \Sigma, \Theta}
$$

The fact that $\mathcal{R}$ is backwards applicable to $S^{i}$ implies that $S^{i a}$ contains some $\square \psi \in$ $\square \Gamma \cup\{\square \varphi\} \cup \square \Pi \cup \Lambda$. In case $\psi=\varphi$ we have, for some partitioning $\left(X^{i}, X^{r}\right)$ of each of the multisets $\Lambda, \Pi, \Gamma, \Delta, \Sigma, \Theta$ :

$$
\begin{aligned}
& S^{i}=\left(\Lambda^{i}, \square \Pi^{i}, \square \Gamma^{i}, \square \varphi \Rightarrow \square \Delta^{i}, \square \Sigma^{i}, \Theta^{i}\right) S_{u}^{i}=\left(\square \Pi^{i}, \Gamma^{i}, \varphi \Rightarrow \Delta^{i}, \square \Sigma^{i}\right) \\
& S^{r}=\left(\Lambda^{r}, \square \Pi^{r}, \square \Gamma^{r} \Rightarrow \square \Delta^{r}, \square \Sigma^{r}, \Theta^{r}\right) \quad S_{u}^{r}=\left(\square \Pi^{r}, \Gamma^{r} \Rightarrow \Delta^{r}, \square \Sigma^{r}\right) .
\end{aligned}
$$

And likewise if $\psi \in \Gamma$. In these cases $S_{u}^{i} / S^{i}$ is an instance of $\mathcal{R}$, thus 1 . holds.

If this is not the case, then $S^{i a}$ contains no formula in $\square \Gamma \cup\{\square \varphi\}$ but there is a $\square \psi \in S^{i a} \cap(\square \Pi \cup \Lambda)$. In case $\psi \in \Pi$, we have, for some partitioning $\left(X^{i}, X^{r}\right)$ of each of the multisets $\Lambda, \Pi, \Delta, \Sigma, \Theta$ with $\psi \in \Pi^{i}$ :

$$
\begin{array}{ll}
S^{i}=\left(\Lambda^{i}, \square \Pi^{i} \Rightarrow \square \Delta^{i}, \square \Sigma^{i}, \Theta^{i}\right) & S_{u}^{i}=\left(\square \Pi^{i} \Rightarrow \Delta^{i}, \square \Sigma^{i}\right) \\
S^{r}=\left(\Lambda^{r}, \square \Pi^{r}, \square \Gamma, \square \varphi \Rightarrow \square \Delta^{r}, \square \Sigma^{r}, \Theta^{r}\right) S_{u}^{r}=\left(\square \Pi^{r}, \Gamma, \varphi \Rightarrow \Delta^{r}, \square \Sigma^{r}\right) .
\end{array}
$$

The only way in which $S_{u}^{i}$ does not consist of boxed formulas is if $\Delta^{i}$ is not empty, and whence $\mathcal{R}$ is an R-rule. Thus it is an WIR-rule, since $\Pi \neq \varnothing$. But this cannot be since $\mathcal{R}$ is not a bad rule. Hence this case cannot occur and $S_{u}^{i}$ consists of boxed formulas only, which gives 2 . It also implies that $S_{u}^{i}=\left(\square \Pi^{i} \Rightarrow \square \Sigma^{i}\right)$ and $S^{i}=\left(\Lambda^{i}, \square \Pi^{i} \Rightarrow\right.$ $\left.\square \Sigma^{i}, \Theta^{i}\right)$. Therefore, for any $\varphi, S_{u}^{i} \cdot(\varphi \Rightarrow) / S^{i} \cdot(\square \varphi \Rightarrow)$ is an instance of $\mathcal{R}$, which proves that the second part of the lemma holds.

If $S^{i a}$ contains no formula in $\square \Gamma \cup\{\square \varphi\} \cup \square \Pi$ but contains a $\square \psi \in \Lambda$, we have, for some partitioning $\left(X^{i}, X^{r}\right)$ of each of the multisets $\Lambda, \Delta, \Sigma, \Theta$ with $\square \psi \in \Lambda^{i}$ :

$$
\begin{array}{ll}
S^{i}=\left(\Lambda^{i} \Rightarrow \square \Delta^{i}, \square \Sigma^{i}, \Theta^{i}\right) & S_{u}^{i}=\left(\Rightarrow \Delta^{i}, \square \Sigma^{i}\right) \\
S^{r}=\left(\Lambda^{r}, \square \Pi, \square \Gamma, \square \varphi \Rightarrow \square \Delta^{r}, \square \Sigma^{r}, \Theta^{r}\right) & S_{u}^{r}=\left(\square \Pi, \Gamma, \varphi \Rightarrow \Delta^{r}, \square \Sigma^{r}\right) .
\end{array}
$$


Thus this is the case in which the antecedent of $S_{u}^{i}$ is empty and for all $\varphi, S_{u}^{i}$. $(\varphi \Rightarrow) / S^{i} \cdot(\square \varphi \Rightarrow)$ is an instance of $\mathcal{R}$. Hence case 3 and the second part of the lemma hold.

We use the abbreviations $f c$ and $f c m$ for focussed and focussed modal, respectively. We will only consider terminating calculi, so that the number of disjuncts in the definitions below is finite.

$$
\begin{aligned}
& \forall^{\mathrm{fr}} p S \sim_{0} \begin{cases}\forall p S & \text { if } S \text { is free and contains a nonboxed formula } \\
\square \forall p S^{\prime} & \text { if } S \text { is free, } S=\square S^{\prime} \text { and } S^{a}=\varnothing \\
\neg \square \neg \forall p S^{\prime} & \text { if } S \text { is free, } S=\square S^{\prime} \text { and } S^{S}=\varnothing \\
\perp & \text { otherwise. }\end{cases} \\
& \forall^{\mathrm{fc}} p S \sim_{0} \forall_{p}^{\text {at }} S \bigvee\{\forall p S \mid R \text { an instance of a fc rule with conclusion } S\} .
\end{aligned}
$$

For an instance $R$ of a focussed modal rule $\mathcal{R}$ backwards applicable to $S$, define

$$
\forall p S \sim_{0} \begin{cases}\square \forall p S_{u} & \text { if } R=S_{u} / S \text { and } \mathcal{R} \text { is a r-rule } \\ \neg \square \neg \forall p S_{u} & \text { if } R=S_{u} / S \text { and } \mathcal{R} \text { is a l-rule. }\end{cases}
$$

For a focussed modal I-rule $\mathcal{R}$ backwards applicable to $S$, define

$$
\begin{aligned}
\forall p R \sim_{0} \bigvee\left\{\square \forall p S_{u}^{i} \mid\right. & \text { for some standard } p \text {-partition } S_{u}^{r} \cdot S_{u}^{i} / S^{r} \cdot S^{i} \text { of an } \\
& \text { instance of } \mathcal{R} \text { with } S=S^{i} \text { and } S_{u}^{i} \prec S \text { and } \mathcal{R} \text { not } \\
& \text { applicable to } \left.S_{u}^{i}\right\} .
\end{aligned}
$$

For a focussed modal r-rule $\mathcal{R}$ backwards applicable to $S$, define

$$
\begin{aligned}
\forall \mathcal{R} S \sim_{0} \bigvee\left\{\neg \square \neg \forall p S_{u}^{i}\right. & \mid \text { for some standard } p \text {-partition } S_{u}^{r} \cdot S_{u}^{i} / S^{r} \cdot S^{i} \text { of an } \\
& \text { instance of } \mathcal{R} \text { with } S=S^{i} \text { and } S_{u}^{i} \prec S \text { and } \mathcal{R} \text { not } \\
& \text { applicable to } \left.S_{u}^{i}\right\} .
\end{aligned}
$$

For a focussed modal I-rule $\mathcal{R}$ not backwards applicable to $S$, define

$$
\begin{gathered}
\forall \stackrel{\mathcal{R}}{p} S \sim_{0} \bigvee\left\{\square \forall p S_{u}^{i} \mid \text { for some standard } p \text {-partition } S_{u}^{r} \cdot S_{u}^{i} / S^{r} \cdot S^{i}\right. \text { of an } \\
\text { instance of } \left.\mathcal{R} \text { with } S=S^{i} \text { and } S_{u}^{i} \prec S\right\} .
\end{gathered}
$$

For a focussed modal r-rule $\mathcal{R}$ not backwards applicable to $S$, define

$$
\begin{gathered}
\forall \stackrel{\mathcal{\mathcal { R }}}{p} S \sim_{0} \bigvee\left\{\neg \square \neg \forall p S_{u}^{i} \mid \text { for some standard } p \text {-partition } S_{u}^{r} \cdot S_{u}^{i} / S^{r} \cdot S^{i}\right. \text { of an } \\
\text { instance of } \left.\mathcal{R} \text { with } S=S^{i} \text { and } S_{u}^{i} \prec S\right\} .
\end{gathered}
$$


In case the calculus contains no WLr-rules or IRE-rules, then define

$$
\begin{aligned}
\forall^{\mathrm{fcm}} p S \sim_{0} & \bigvee\{\forall p h \mid R \text { an instance of a fcm rule with conclusion } S\} \vee \\
& \bigvee\{\forall \stackrel{\mathcal{R}}{p} S \mid \mathcal{R} \text { a fcm rule backwards applicable to } S\} \vee \\
& \bigvee\{\forall \stackrel{\mathcal{\mathcal { R }}}{p} S \mid \mathcal{R} \text { a fcm rule not backwards applicable to } S\} .
\end{aligned}
$$

And otherwise define

$$
\begin{aligned}
\forall^{\mathrm{fcm}} p S \sim_{0} & \forall^{\mathrm{em}} p S \vee \\
& \bigvee\{\forall p s \mid R \text { an instance of a fcm rule with conclusion } S\} \vee \\
& \bigvee\{\forall p s \mid \mathcal{R} \text { a fcm rule backwards applicable to } S\} \vee \\
& \bigvee\left\{\forall^{\mathcal{R}} S \mid \mathcal{R} \text { a fcm rule not backwards applicable to } S\right\},
\end{aligned}
$$

where

$$
\forall^{\mathrm{em}} p \sim_{0} \begin{cases}\bigvee\{\neg \square \neg \forall p(\Gamma \Rightarrow) \mid(\square \Gamma \Rightarrow) \subseteq S\} & \text { if the calculus contains a } \\ \bigvee\{\square \forall p(\Rightarrow \Delta) \mid(\Rightarrow \square \Delta) \subseteq S\} & \text { WLr-rule. } \\ & \text { if the calculus contains an } \\ \perp & \text { IRE-rule. } \\ & \text { otherwise. }\end{cases}
$$

The standard assignment in this modal setting is defined as $\forall p S \sim_{0} \top$ if $S$ is an axiom, and in all other cases:

$$
\forall p S \sim_{0} \forall^{\mathrm{fr}} p S \vee \forall^{\mathrm{fc}} p S \vee \forall^{\mathrm{fcm}} p S .
$$

Because in the case of focussed modal rules, the uniform interpolants have become more complicated, we need to reformulate the sufficient conditions for uniform interpolation in the following way. Given a calculus and an interpolant assignment, we distinguish three properties.

(IP) If $S$ is not free and all sequents lower than $S$ satisfy the interpolant properties, then $S$ satisfies the independent interpolant property.

(DPB) If all sequents lower than $S$ satisfy the interpolant properties and $S$ has a derivation which last inference is an instance of a rule $\mathcal{R}$, then for every partition $\left(S^{r}, S^{i}\right)$ of $S$ such that $S^{i}$ is not empty and $\mathcal{R}$ is backwards applicable to $S^{i}$, the dependent interpolant property holds.

(DPN) If all sequents lower than $S$ satisfy the interpolant properties and $S$ has a derivation which last inference is an instance of a rule $\mathcal{R}$, then for every partition $\left(S^{r}, S^{i}\right)$ of $S$ such that $S^{i}$ is not empty and $\mathcal{R}$ is not backwards applicable to $S^{i}$, the dependent interpolant property holds.

Property (IP) holds if it holds for all sequents $S$, and similarly for (DPB) and (DPN). As in Lemma 2 it is not hard to show that the above properties are sufficient for having uniform interpolation: 
Lemma 14 If a logic $L$ has a terminating calculus for which there exists an interpolant assignment such that (IP), (DPB), (DBN) and $(F P)$ hold, then all sequents satisfy the interpolant properties.

\subsection{Properties of focussed modal rules}

In the following lemmas,

$$
\frac{\square S_{1} \cdot S_{0}}{S_{2} \cdot \square S_{1} \cdot \square S_{0}} R
$$

will always be an instance of a focussed modal rule $\mathcal{R}$, and $S_{u}=\square S_{1} \cdot S_{0}$ denotes the upper sequent and $S_{l}=S_{2} \cdot \square S_{1} \cdot \square S_{0}$ the lower sequent of $R$.

One of the properties that we use to prove that our calculi have uniform interpolation is that of being balanced. Given a calculus, a rule $\mathcal{R}_{\mathrm{rx}}$ or $\mathcal{R}_{\mathrm{Ix}}$ is covered if the calculus contains an Lrx-rule or an IRx-rule. A calculus is balanced if it does not contain bad rules, every rule is covered, and if it contains a Wr-rule or an IE-rule, that is the only modal rule it contains. Observe that this implies that if a balanced calculus contains a Wr-rule or an IE-rule, it is the unique modal rule in the calculus and a WLr-rule or an IRE-rule, respectively.

Lemma 15 If $\left(S_{u} / S_{l}\right)$ is an instance of a focussed modal $\mathrm{r}$-rule $\mathcal{R}$ in a balanced calculus, then $S_{u} \cdot(\varphi \Rightarrow) \vdash S_{l} \cdot(\square \varphi \Rightarrow)$ for any formula $\varphi$.

Proof As the calculus is balanced, $\mathcal{R}=\mathcal{R}_{\mathrm{xr}}$ is covered, meaning that the calculus contains an Lxr-rule or an IRx-rule. If the calculus contains an Lxr-rule, then it can be applied to $S_{u} \cdot(\varphi \Rightarrow)$ to obtain $S_{l} \cdot(\square \varphi \Rightarrow)$. If the calculus does not contain an Lxr-rule, then $\mathcal{R}$ is not an L-rule, and thus $S_{0}^{a}$ is empty. Therefore the IRx-rule can be applied to $S_{u} \cdot(\varphi \Rightarrow)$ to obtain $S_{l} \cdot(\square \varphi \Rightarrow)$.

Lemma 16 If $\left(S_{u} / S_{l}\right)$ is an instance of a focussed modal I-rule $\mathcal{R}$ in a balanced calculus for a logic, then $S_{u} \cdot(\varphi \Rightarrow) \vdash S_{l} \cdot(\neg \square \neg \varphi \Rightarrow)$ for any formula $\varphi$.

Proof As logics contain CPC by assumption, $S_{u} \cdot(\varphi \Rightarrow) \vdash S_{u} \cdot(\Rightarrow \neg \varphi)$. As the calculus is balanced, $\mathcal{R}=\mathcal{R}_{\mathrm{Ix}}$ is covered, meaning that the calculus contains an IRxrule or an LrX-rule. In the first case, the IRx-rule can be applied to $S_{u} \cdot(\Rightarrow \neg \varphi)$ to obtain $S_{l} \cdot(\Rightarrow \square \neg \varphi)$. This last sequent derives the desired $S_{l} \cdot(\neg \square \neg \varphi \Rightarrow)$. In the second case, $\mathcal{R}$ is not an R-rule, thus $S_{0}^{S}$ is empty. Therefore the LrX-rule can be applied to $S_{u} \cdot(\Rightarrow \neg \varphi)$ to obtain $S_{l} \cdot(\Rightarrow \square \neg \varphi)$.

Lemma 17 In any balanced terminating calculus consisting offocussed and focussed modal rules (IP) holds.

Proof If $S$ is an axiom, it is derivable and has to be focussed, and is therefore closed under weakening at the left. The independent interpolant property follows from this. Therefore assume $S$ is not an axiom and not free. We have to show, under the assumption that all sequents $\prec$-lower than $S$ satisfy the interpolant properties, that

$$
\vdash S^{a}, \forall^{\mathrm{fr}} p S \Rightarrow S^{S} \vdash S^{a}, \forall^{\mathrm{fc}} p S \Rightarrow S^{S} \vdash S^{a}, \forall^{\mathrm{fcm}} p S \Rightarrow S^{S} .
$$


This means that for every disjunct $\chi$ of $\forall^{\mathrm{fr}} p S, \forall^{\mathrm{fc}} p S$ and $\forall^{\mathrm{fcm}} p S$ we have to prove that

$$
\vdash S^{a}, \chi \Rightarrow S^{s}
$$

First consider a disjunct $\chi$ of $\forall^{\mathrm{fc}} p S$. Thus $\chi=\forall_{p}^{\text {at }} S$ or $\chi=\forall p$ for an instance $R$ of a focussed rule. The first case is treated as in the proof of Lemma 2, and the second case as in the proof of Lemma 7, using the assumption that the premisses of $R$ satisfy the interpolant properties.

Next suppose $\chi$ is a disjunct of $\forall^{\mathrm{fr}} p$. Since $S$ is not free, $\chi=\perp$ and (5) trivially holds. The case that $\chi$ is a disjunct of $\forall^{\mathrm{fcm}} p S$ remains. We distinguish the following four cases.

First, we consider disjuncts $\chi$ of $\forall^{\mathrm{em}} p S$. The case that $\chi=\perp$ is trivial. We treat the case that $\chi=\neg \square \neg \forall p(\Gamma \Rightarrow)$, where $(\square \Gamma \Rightarrow) \subseteq S$ and the calculus contains a WLrrule $\mathcal{R}$. The disjuncts of $\forall^{\mathrm{em}} p S$ of the form $\square \forall p(\Rightarrow \Delta)$ can be treated in a similar way. As $\vdash \Gamma \Rightarrow \neg \forall p(\Gamma \Rightarrow)$ and $\mathcal{R}$ is an Lr-rule, it can be applied to $\Gamma \Rightarrow \neg \forall p(\Gamma \Rightarrow)$ to obtain $\vdash S^{a} \Rightarrow \square \neg \forall p(\Gamma \Rightarrow), S^{s}$. This gives $\vdash S^{a}, \chi \Rightarrow S^{s}$ which is what had to be shown.

Second, suppose $\chi=\square \forall p S_{u}, S=S_{l}$ and $S_{u} / S_{l}$ is an instance of a fcm r-rule. Thus $\vdash S_{u} \cdot\left(\forall p S_{u} \Rightarrow\right)$ by assumption. Lemma 15 implies (5). The case $\chi=\neg \square \neg \forall p S_{u}$, where $S=S_{l}$ and $S_{u} / S_{l}$ is an instance of a fcm l-rule, follows in a similar way from Lemma 16.

Third, $\chi=\neg \square \neg \forall p S_{u}$, where $S_{u} \prec S$ and for some fcm r-rule $\mathcal{R}$ that is backwards applicable to $S$ there are sequents $S^{\prime}, S_{u}^{\prime}$ such that $S_{u}^{\prime} \cdot S_{u} / S^{\prime} \cdot S$ is a standard $p$-partition of an instance of $\mathcal{R}$ and $\mathcal{R}$ is not applicable to $S_{u}$. This implies that $\vdash S_{u} \cdot\left(\forall p S_{u} \Rightarrow\right)$, and thus $\vdash S_{u} \cdot\left(\Rightarrow \neg \forall p S_{u}\right)$. As $S_{u} / S$ is not an instance of $\mathcal{R}, \vdash S \cdot\left(\Rightarrow \square \neg \forall p S_{u}\right)$ holds by Lemma 13, which is what had to be shown. The case that $\chi$ is of the form $\square \forall p S_{u}$ and $\mathcal{R}$ is a fcm I-rule backwards applicable to $S$ is similar.

In the fourth case, $\chi=\square \forall p S_{u}$, where $S_{u} \prec S$ and for some fcm l-rule $\mathcal{R}$ that is not backwards applicable to $S$ there are sequents $S_{u}^{\prime}, S^{\prime}$ such that $S_{u}^{\prime} \cdot S_{u} / S^{\prime} \cdot S$ is a standard $p$-partition of an instance of $\mathcal{R}$. We have $\vdash S_{u} \cdot\left(\forall p S_{u} \Rightarrow\right)$ because (IP) holds for $S_{u}$. As $S_{u} / S$ is not an instance of $\mathcal{R}$, it follows from Lemma 13 that $\vdash S \cdot\left(\square \forall p S_{u} \Rightarrow\right)$, which gives (5). The case that $\chi$ is of the form $\neg \square \neg \forall p S_{u}$ and $\mathcal{R}$ is a fcm r-rule not backwards applicable to $S$ is similar.

Lemma 18 In any balanced terminating calculus consisting offocussed and focussed modal rules $(D P B)$ holds.

Proof Since axioms receive $T$ as interpolant assignment, (DPB) holds for axioms. Therefore consider a sequent $S$ that is not an axiom, that has a derivation which last inference is an instance $R$ of a rule $\mathcal{R}$. Let $\left(S^{r}, S^{i}\right)$ be a $p$-partition of $S$ such that $S^{i}$ is not empty and $\mathcal{R}$ is backwards applicable to $S^{i}$. We have to show that

$$
\vdash S^{r} \cdot\left(\Rightarrow \forall p S^{i}\right)
$$

For focussed rules the proof of (6) is similar to the proof of Lemma 8. Therefore suppose $\mathcal{R}$ is a focussed modal rule, $R=S_{u} / S$, and $\mathcal{R}$ is backwards applicable to $S^{i}$ 
and consider the standard $p$-partition of $\mathcal{R}$. By the assumption that all sequents lower than $S$ satisfy the interpolant properties, $\vdash S_{u}^{r} \cdot\left(\Rightarrow \forall p S_{u}^{i}\right)$.

First, suppose $S_{u}^{i} / S^{i}$ is an instance of $\mathcal{R}$. Thus $\square \forall p S_{u}^{i}$ is a disjunct of $\forall p S^{i}$ if $\mathcal{R}$ is an r-rule and $\neg \square \neg \forall p S_{u}^{i}$ is a disjunct of $\forall p S^{i}$ if $\mathcal{R}$ is an l-rule. Since $\vdash S_{u}^{r} \cdot\left(\Rightarrow \forall p S_{u}^{i}\right)$, for (6) it therefore suffices to show that

$$
\begin{array}{ll}
S_{u}^{r} \cdot\left(\Rightarrow \forall p S_{u}^{i}\right) \vdash S^{r} \cdot\left(\Rightarrow \square \forall p S_{u}^{i}\right) & \text { if } \mathcal{R} \text { is an r-rule, } \\
S_{u}^{r} \cdot\left(\Rightarrow \forall p S_{u}^{i}\right) \vdash S^{r} \cdot\left(\Rightarrow \neg \square \neg \forall p S_{u}^{i}\right) & \text { if } \mathcal{R} \text { is an I-rule. }
\end{array}
$$

If $\mathcal{R}$ is an r-rule, there are three possibilities: (1) $\mathcal{R}$ is an R-rule, (2) $\mathcal{R}$ is not an R-rule but it is a E-rule and $S_{u}^{r s}$ consists of boxed formulas only, (3) $\mathcal{R}$ is not an R-rule and not a E-rule and $S_{u}^{r s}$ is empty. In all three cases $\mathcal{R}$ can be applied to $S_{u}^{r} \cdot\left(\Rightarrow \forall p S_{u}^{i}\right)$ to obtain $S^{r} \cdot\left(\Rightarrow \square \forall p S_{u}^{i}\right)$.

We turn to the case that $S_{u}^{i} / S^{i}$ is not an instance of $\mathcal{R}$. We distinguish according to cases 2-4 in Lemma 13, one of which has to hold, and use the notation from the proof of that lemma.

2. We start with the case that $S_{u}^{i}$ consists of boxed formulas only. We only treat the case that $\mathcal{R}$ is an I-rule, say $\mathcal{R}=\mathcal{R}_{\mathrm{Ix}}$, the other case being similar. Thus we have

$$
\begin{array}{ll}
S^{i}=\left(\Lambda^{i}, \square \Pi^{i} \Rightarrow \square \Sigma^{i}, \Theta^{i}\right) & S_{u}^{i}=\left(\square \Pi^{i} \Rightarrow \square \Sigma^{i}\right) \\
S^{r}=\left(\Lambda^{r}, \square \Pi^{r}, \square \Gamma, \square \varphi \Rightarrow \square \Delta, \square \Sigma^{r}, \Theta^{r}\right) & S_{u}^{r}=\left(\square \Pi^{r}, \Gamma, \varphi \Rightarrow \Delta, \square \Sigma^{r}\right) .
\end{array}
$$

If $\Pi \neq \varnothing$, then $\mathcal{R}$ is a $\mathrm{Wl}$-rule, and as every rule is covered the calculus contains a WIR-rule or a WLr-rule. Since the first rule is bad, the second rule has to be present. But that contradicts the fact that if a WLr-rule is present it is the unique fcm rule. This proves that $\Pi=\varnothing$, and thus $S_{u}^{i a}=\varnothing$. As $S_{u}^{i}$ is not empty, $\mathcal{R}$ is an IE-rule, and thus the only modal rule, because the calculus is balanced. Observe that whence all disjuncts of $\forall p S_{u}^{i}$ are boxed formulas or $\perp$, so $\forall p S_{u}^{i}$ is equivalent to a formula $\bigvee_{i=1}^{n} \square \varphi_{i}$. Hence $\vdash S_{u}^{r} \cdot\left(\Rightarrow \square \varphi_{1}, \ldots, \square \varphi_{n}\right)$. An application of $\mathcal{R}$ to that sequent gives $\vdash S^{r} \cdot\left(\Rightarrow \square \varphi_{1}, \ldots, \square \varphi_{n}\right)$, which implies $\vdash S^{r} \cdot\left(\Rightarrow \forall p S_{u}^{i}\right)$.

Therefore, to prove (6) it suffices to show that $\forall p S_{u}^{i}$ implies $\forall p S^{i}$, which means, that any disjunct of $\forall p S_{u}^{i}$ implies $\forall p S^{i}$. Since $\mathcal{R}$ is the only modal rule the only disjuncts that need to be considered are $\forall^{\mathrm{em}} p S_{u}^{i}$ and the disjuncts of $\forall p S_{u}^{\overline{\mathcal{R}}}$. For disjuncts of $\forall^{\mathrm{em}} p S_{u}^{i}$ it is clear that they are disjuncts of $\forall^{\mathrm{em}} p S^{i}$ since $S_{u}^{i} \subseteq S^{i}$.

Therefore consider a disjunct $\square \forall p S_{2}$ of $\forall \bar{p} S_{u}^{i}$. Thus there is a standard $p$-partition $S_{3} \cdot S_{2} / S_{1} \cdot S_{u}^{i}$ of an instance of $\mathcal{R}$ with $S_{2} \prec S_{u}^{i}$ and $S_{2}^{a}=S_{u}^{i a}=\varnothing$. Note that $S_{3} \cdot S_{2} / S_{1} \cdot S^{i}$ is the standard $p$-partition of an instance of $\mathcal{R}$ as well. And as $\mathcal{R}$ is backwards applicable to $S^{i}$ and is not applicable to $S_{2}, \square \forall p S_{2}$ is a disjunct of $\forall p S^{i}$ and thus of $\forall p S^{i}$.

This completes the proof that any disjunct $\forall p S_{u}^{i}$ is a disjunct of $\forall p S^{i}$, and thereby finishes case 2 of Lemma 13.

3. Suppose $S_{u}^{i} / S^{i}$ is not an instance of $\mathcal{R}, S_{u}^{i}$ contains nonboxed formulas, $S_{u}^{i a}=\varnothing$, and $\mathcal{R}$ is an I-rule. By Lemma 13 we have: 


$$
\begin{array}{ll}
S^{i}=\left(\Lambda^{i} \Rightarrow \square \Delta^{i}, \square \Sigma^{i}, \Theta^{i}\right) & S_{u}^{i}=\left(\Rightarrow \Delta^{i}, \square \Sigma^{i}\right) \\
S^{r}=\left(\Lambda^{r}, \square \Pi, \square \Gamma, \square \varphi \Rightarrow \square \Delta^{r}, \square \Sigma^{r}, \Theta^{r}\right) & S_{u}^{r}=\left(\square \Pi, \Gamma, \varphi \Rightarrow \Delta^{r}, \square \Sigma^{r}\right) .
\end{array}
$$

As $\Delta^{i}$ is not empty, $\mathcal{R}$ is an IR-rule. Thus we can apply $\mathcal{R}$ to $S_{u}^{r} \cdot\left(\Rightarrow \forall p S_{u}^{i}\right)$, which is derivable, and establish that $\vdash S^{r} \cdot\left(\Rightarrow \square \forall p S_{u}^{i}\right)$. Since $\mathcal{R}$ is not applicable to $S_{u}^{i}$ but is backwards applicable to $S^{i}, \square \forall p S_{u}^{i}$ is a disjunct of $\forall p S^{\mathcal{R}}$ and thereby of $\forall p S^{i}$, which implies $\vdash S^{r} \cdot\left(\Rightarrow \forall p S^{i}\right)$.

4. Similar to case 3 and therefore left to the reader.

Lemma 19 In any balanced terminating calculus consisting offocussed and focussed modal rules (DPN) holds.

Proof Consider a $p$-partition $\left(S^{r}, S^{i}\right)$ of a derivable sequent $S$, where the last rule in the derivation is $\mathcal{R}$, which is not backwards applicable to $S^{i}$ (whence $S^{i} \neq S$ ) and $S^{i}$ is not empty. We have to show that under the assumption that all sequents lower than $S$ satisfy the interpolant properties,

$$
\vdash S^{r} \cdot\left(\Rightarrow \forall p S^{i}\right)
$$

For $\mathcal{R}$ a focussed rule (or axiom), the proof of (7) is analogous to the proof of Lemma 10. It remains to consider the case that $\mathcal{R}$ is a focussed modal rule and the last step of the derivation of $S$ consists of instance $R=S_{u} / S$, which has standard $p$-partition $S_{u}^{r} \cdot S_{u}^{i} / S^{r} \cdot S^{i}$. We distinguish the case that $S_{u}^{i} \prec S^{i}$ and that $S_{u}^{i}=S^{i}$. In the first case, $\square \forall p S_{u}^{i}$ is a disjunct of $\forall \overline{\mathcal{R}} S^{i}$ and thus of $\forall p S^{i}$ if $\mathcal{R}$ is an I-rule and $\neg \square \neg \forall p S_{u}^{i}$ is a disjunct of $\forall p S^{i}$ if $\mathcal{R}$ is an r-rule. We only treat the last case, where $\mathcal{R}=\mathcal{R}_{\mathrm{xr}}$. For (7) it suffices to show that $\vdash S^{r} \cdot\left(\Rightarrow \neg \square \neg \forall p S_{u}^{i}\right)$.

We have $\vdash S_{u}^{r} \cdot\left(\Rightarrow \forall p S_{u}^{i}\right)$ as $S_{u}$ is derivable, and thus $S_{u}^{r} \cdot\left(\neg \forall p S_{u}^{i} \Rightarrow\right)$ is derivable as well. As the calculus is balanced, it contains an Lxr-rule or an IRx-rule. In the first case, that rule can be applied to $S_{u}^{r} \cdot\left(\neg \forall p S_{u}^{i} \Rightarrow\right)$. It yields $\vdash S^{r} \cdot\left(\square \neg \forall p S_{u}^{i} \Rightarrow\right)$, from which $\vdash S^{r} \cdot\left(\Rightarrow \neg \square \neg \forall p S_{u}^{i}\right)$ follows. In the second case, $\mathcal{R}$ is not an L-rule, and thus the IRx-rule can be applied to $S_{u}^{r} \cdot\left(\neg \forall p S_{u}^{i} \Rightarrow\right)$, to obtain $\vdash S^{r} \cdot\left(\square \neg \forall p S_{u}^{i} \Rightarrow\right)$.

The case that $S_{u}^{i}=S^{i}$ remains. We only treat the case that $\mathcal{R}$ is an r-rule. Given that $\mathcal{R}$ is of the form (4), this implies that $S^{i}$ is part of $\square S_{1}$. As $\mathcal{R}$ is not backwards applicable to $S^{i}, S^{i}=S_{u}^{i}=(\square \Gamma \Rightarrow)$ and $\mathcal{R}$ is a Wr-rule. Because the calculus is balanced, it is the only modal rule and a WLr-rule. This implies that $\forall p S^{i}=\forall p S_{u}^{i}$ is a nonempty disjunction of the form $\bigvee_{i=1}^{n} \neg \square \neg \varphi_{i}$. Since $\vdash S_{u}^{r} \cdot\left(\Rightarrow \forall p S^{i}\right)$, also $S_{u}^{r} \cdot\left(\square \neg \varphi_{1}, \ldots, \square \neg \varphi_{n} \Rightarrow\right)$ is derivable. As $\mathcal{R}$ is an W-rule, it can be applied to this sequent to obtain $\vdash S^{r} \cdot\left(\square \neg \varphi_{1}, \ldots, \square \neg \varphi_{n} \Rightarrow\right)$, which implies $\vdash S^{r} \cdot\left(\Rightarrow \forall p S^{i}\right)$.

Lemma 20 If a terminating calculus consists of focussed and focussed modal rules only, then (FP) holds.

Proof Let $S$ be a free sequent and assume that all sequents $\prec-l o w e r$ than $S$ satisfy the interpolant properties. We have to show that $S$ satisfies the independent interpolant 
property, that is, that $S^{a}, \forall p S \Rightarrow S^{S}$ is derivable. It suffices to show that for all disjuncts $\chi$ of $\forall^{\mathrm{fr}} p S, \forall^{\mathrm{fc}} p S, \forall^{\mathrm{fcm}} p S$ :

$$
\vdash S^{a}, \chi \Rightarrow S^{s}
$$

If $\chi$ is a disjunct of $\forall^{\mathrm{fc}} p S$ or a disjunct of $\forall^{\mathrm{fr}} p S$ of the form $\forall_{p}^{\varnothing} S$, then the proof is similar to the proof of Lemma 11. If it is a disjunct of $\forall^{\mathrm{fr}} p S$ of the form $\chi=\neg \square \neg \forall p S^{\prime}$ for some $S^{\prime}=(\Gamma \Rightarrow)$, then we use that $\Gamma, \forall p S^{\prime} \Rightarrow$ is derivable by assumption. As $S=\square S^{\prime}$ is free, the calculus contains no l-rules, and because it is balanced it therefore contains an Lr-rule (if it contains no modal rules, the calculus is just G3p). This rule can be applied to obtain $\vdash \square \Gamma \Rightarrow \square \neg \forall p S^{\prime}$, and thereby $\vdash(\square \Gamma, \chi \Rightarrow)$. The case that $\chi=\square \forall p S^{\prime}$ for some $S^{\prime}=(\Rightarrow \Delta)$ is analogous.

Thus $\forall^{\mathrm{fcm}} p S$ remains, where $\chi$ has to be a disjunct of $\forall \bar{p} S$ for some rule $\mathcal{R}$ such that $S_{u}^{r} \cdot S_{u}^{i} / S^{r} \cdot S^{i}$ is a standard $p$-partition of an instance of it, where $S=S^{i}$. We distinguish the case that $S_{u}^{i} \prec S^{i}$ and that $S_{u}^{i}=S^{i}$. In the first case we treat the case that $\mathcal{R}$ is an r-rule, the case that it is an I-rule is similar. Thus $\mathcal{R}=\mathcal{R}_{\mathrm{xr}}$ and $\chi=\neg \square \neg \forall p S_{u}^{i}$, and $S_{u}^{i s}$ is empty as $\mathcal{R}$ is not backwards applicable to $S^{i}$. Since all sequents $\prec-$ lower than $S$ satisfy the interpolant properties, $S_{u}^{i} \cdot\left(\forall p S_{u}^{i} \Rightarrow\right)$ is derivable. Because $\mathcal{R}$ is an r-rule applicable to $S_{u}^{r} \cdot S_{u}^{i}$ and $S_{u}^{i s}$ is empty, it is applicable to $S_{u}^{i} \cdot\left(\Rightarrow \neg \forall p S_{u}^{i}\right)$. This yields $\vdash S^{i} \cdot\left(\Rightarrow \square \neg \forall p S_{u}^{i}\right)$, from which $\vdash S^{i} \cdot\left(\neg \square \neg \forall p S_{u}^{i} \Rightarrow\right)$ follows.

The case that $S_{u}^{i}=S^{i}$ remains. We only treat the case that $\mathcal{R}$ is an I-rule, the other case is treated in the last case of Lemma 19, to which this case is analogous. Given that $\mathcal{R}$ is of the form (4), this implies that $S^{i}$ is part of $\square S_{1}$. And as $\mathcal{R}$ is not backwards applicable to $S^{i}, S^{i}=S_{u}^{i}=(\Rightarrow \square \Delta)$ and $\mathcal{R}$ is an E-rule. Since the calculus is balanced, it therefore is the only modal rule and of the form $\mathcal{R}_{\operatorname{IREx}}$. This implies that $\forall p S^{i}=\forall p S_{u}^{i}$ is a nonempty disjunction of the form $\bigvee_{i=1}^{n} \square \varphi_{i}$. Since $\vdash S_{u}^{r} \cdot\left(\Rightarrow \forall p S^{i}\right)$, also $S_{u}^{r} \cdot\left(\Rightarrow \square \varphi_{1}, \ldots, \square \varphi_{n}\right)$ is derivable. As $\mathcal{R}$ is an E-rule, it can be applied to this sequent to obtain $\vdash S^{r} \cdot\left(\Rightarrow \square \varphi_{1}, \ldots, \square \varphi_{n}\right)$, which implies $\vdash S^{r} \cdot\left(\Rightarrow \forall p S^{i}\right)$.

Since any calculus that consists of focussed and focussed modal rules is closed under weakening, the previous lemmas imply the following two theorems.

Theorem 4 For any balanced terminating calculus that consists of focussed and focussed modal rules, (IP), (DPB), (DBN) and (FP) hold for the standard interpolant assignment.

Theorem 5 A logic $\mathrm{L} \supseteq \mathrm{CPC}$ with a balanced terminating calculus that consists of focussed and focussed modal rules has uniform interpolation.

\section{Applications}

In order to apply Theorem 5 it first has to be established that many standard calculi for modal logics are terminating. For this we use the following weight function on modal 
formula from [1]: $\varphi \prec \psi \equiv_{\text {def }} w(\varphi)<w(\psi)$, where

$$
\begin{aligned}
& w(p)=w(\perp)=1 \\
& w(\varphi \circ \psi)=w(\varphi)+w(\psi)+1 \quad \circ \in\{\wedge, \vee, \rightarrow\} \\
& w(\square \varphi)=w(\neg \varphi)=w(\varphi)+1 .
\end{aligned}
$$

The weight $w(\Gamma)$ of a multiset $\Gamma$ is the sum of the weights of the formula occurrences in $\Gamma$, and the weight $w(S)$ of a sequent is $w\left(S^{a} \cup S^{s}\right)$. It is easy to see that calculi consisting of G3p plus some focussed modal rules are terminating with respect to this order.

Next, we want to guarantee that our calculi G3K and G3KD are indeed calculi for the modal logics $\mathrm{K}$ and KD. To that end we prove that the restricted focussed modal rules $\mathcal{R}$ are in fact not weaker than their full version $\mathcal{R}^{+}$, which are defined in the first part of Sect. 5 .

Lemma 21 For any extension $\mathrm{G}$ of $\mathrm{G} 3 \mathrm{p}$ that consists of focussed and focussed modal rules, the calculus $\mathrm{G}$ extended by $\mathcal{R}$ is equal to $\mathrm{G}$ extended by $\mathcal{R}^{+}$.

Proof We have to show that for any calculus $G$ that consists of focussed rules and focussed modal rules $\mathcal{R}$ for which $\mathcal{R}^{+}=\mathcal{R}$, for any number of rules $\mathcal{R}_{1}, \ldots, \mathcal{R}_{n}$ such that $\mathcal{R}_{i} \neq \mathcal{R}_{i}^{+}$for all $i, \mathrm{G}$ extended by $\mathcal{R}_{1}, \ldots, \mathcal{R}_{n}$ is equal to $\mathrm{G}$ extended by $\mathcal{R}_{1}^{+}, \ldots, \mathcal{R}_{n}^{+}$. Let $\vdash$ denote derivability in $\mathrm{G}$ extended by $\mathcal{R}_{1}, \ldots, \mathcal{R}_{n}$. It suffices to prove with induction to the length $d$ of derivations that if $\vdash S$, then $\vdash S \cdot(\Rightarrow \varphi)$ and $\vdash S \cdot(\varphi \Rightarrow)$ for any sequent $S$ and any formula $\varphi$. We prove both cases simultaneously and use a subinduction to $\varphi$, but we only treat the case $(\Rightarrow \varphi)$ explicitly. Let $S^{\prime}=$ $(\Rightarrow \varphi)$.

If $S$ is an instance of an axiom, then because the axiom has to be focussed, $S \cdot S^{\prime}$ is an instance of that axiom too. Suppose that $S$ has a proof of depth $d>1$. If the last rule is not one of $\mathcal{R}_{i}$, then the derivability of $S \cdot S^{\prime}$ follows from the induction hypothesis and the fact that the rules of $G$ are closed under weakening. Therefore suppose it is an instance of one of the $\mathcal{R}_{h}$, where $S=S_{2} \cdot \square S_{1} \cdot \square S_{0}$ and the premiss is $\square S_{1} \cdot S_{0}$. Thus for $i=0$ or $i=1$ (or both): $S_{i}^{a}$ or $S_{i}^{S}$ contains a meta-multiset. We show that $\vdash S \cdot S^{\prime}$ with induction to $w(\varphi)$.

If $\varphi$ is an atom, then $\vdash S \cdot S^{\prime}$ follows by an application of $\mathcal{R}_{h}$ to $\square S_{1} \cdot S_{0}$. Therefore suppose that $\varphi$ is not an atom and that $\vdash S \cdot S^{\prime \prime}$ has been shown for all $S^{\prime \prime}=(\Rightarrow \psi)$ and $S^{\prime \prime}=(\psi \Rightarrow)$ with $w(\psi)<w(\varphi)$. If $\varphi$ is a conjunction $\psi \wedge \chi \in S^{a}$, then $\vdash S \cdot\left(S^{\prime a} \backslash\{\chi\}, \psi, \chi \Rightarrow S^{\prime s}\right)$ by the induction hypothesis. Thus an application of the left conjunction rule implies that $\vdash S \cdot S^{\prime}$. Likewise for $\psi \wedge \chi \in S^{S}$ and for all other connectives. Finally, suppose $\varphi=\square \psi$. Since $\mathcal{R}_{h}$ is not equal to $\mathcal{R}_{h}^{+}$, there is an $x \in\{a, s\}$ such that $S_{2}^{x}$ ranges over multisets of atoms, and either $S_{1}^{x}$ or $S_{0}^{x}$ contains a multiset. Thus $\mathcal{R}$ is an R-rule or an E-rule. We treat the case that $x=s$. By the induction hypothesis the following two sequents are derivable.

$$
\square S_{1} \cdot S_{0} \cdot(\Rightarrow \psi) \quad \square S_{1} \cdot S_{0} \cdot(\Rightarrow \square \psi)
$$

In case $\mathcal{R}$ is an R-rule we apply it to the first sequent to establish $\vdash S \cdot S^{\prime}$ and if $\mathcal{R}$ is an E-rule we apply it to the second sequent. 
Despite the above lemma, not all calculi consisting of focussed and focussed modal rules correspond to a logic, because the cut rule is not always admissible in them. An example is the logic G3p $+\mathcal{R}_{W L r}$. Below it is explained that contraction is not admissible in it. Hence neither is the cut rule.

For the application of Theorem 5 the next issue that needs to be addressed is which of the calculi that consist of G3p (for the modal language) plus some focussed modal rules are balanced. There are 32 calculi that contain exactly one focussed modal rule, for which calculi it is easy to establish whether they are balanced.

Theorem 6 Every calculus that consists of $\mathrm{G} 3 \mathrm{p}$ plus a single rule $\mathcal{R}_{\mathrm{x}}$, where $\mathrm{x}$ contains $\mathrm{Lr}$ or IR but not LrE and not WIR, has uniform interpolation.

For calculi that contain more than one focussed modal rule the situation is more complex. Here we restrict ourselves to calculi with two focussed modal rules where one is an extension of $\mathcal{R}_{\mathrm{K}}$. The following theorem follows immediately from the properties of balanced calculi.

Theorem 7 If $\mathcal{R}_{1}=\mathcal{R}_{\mathrm{Lrx}}$ and $\mathcal{R}_{2}$ are two different fcm rules and $\mathrm{G} 3 \mathrm{p}$ plus these two rules is a balanced caculus, then either $\mathrm{x}$ is empty or equal to $\mathrm{R}$ and $\mathcal{R}_{2}$ is one of $\mathcal{R}_{\mathrm{I}}, \mathcal{R}_{\mathrm{IR}}, \mathcal{R}_{\mathrm{r}}$. Thus for these rules the calculus has uniform interpolation.

Similar results can be obtained for extensions of IR-rules, as well as for calculi with more than two focussed modal rules.

The terminating calculus G3K for the modal logic K consists of G3p (for the modal language) extended by the rule $\mathcal{R}_{\mathrm{K}}=\mathcal{R}_{\mathrm{Lr}}$ below, and similarly for the calculus G3D. G3KD stands for the calculus G3K extended by $\mathcal{R}_{\mathrm{D}}=\mathcal{R}_{\mathrm{LI}}$.

$$
\frac{\Gamma \Rightarrow \varphi}{\Gamma^{\prime}, \square \Gamma \Rightarrow \square \varphi, \Delta} \mathcal{R}_{\mathrm{K}} \quad \frac{\Gamma, \varphi \Rightarrow}{\Gamma^{\prime}, \square \Gamma, \square \varphi \Rightarrow \Delta} \mathcal{R}_{\mathrm{D}}
$$

The versions $\mathcal{R}_{\mathrm{K}}^{-}$and $\mathcal{R}_{\mathrm{D}}^{-}$of the above rules in which $\Gamma^{\prime}$ ranges over multisets of atoms are focussed modal rules, and in Lemma 21 it is shown that G3p $+\mathcal{R}_{\mathrm{K}}^{-}$and $\mathrm{G} 3 p+\mathcal{R}_{\mathrm{K}}^{-}+\mathcal{R}_{\mathrm{D}}^{-}$are equivalent to G3K and G3KD, respectively. As a corollary of Theorem 6 and Theorem 7 we therefore obtain the following results, where the result about $\mathrm{K}$ was first proved by Bílková [1].

Theorem $8 \mathrm{~K}$ and $\mathrm{KD}$ have uniform interpolation.

S4 is known to have no uniform interpolation [3], and the same holds for K4 [1]. This leads to the following observation.

Theorem 9 Neither K4 nor S4 is sound and complete with respect to a balanced terminating extension of G3p by focussed and focussed modal rules.

The theorem implies that the calculus $\mathrm{G} 3 p+\mathcal{R}_{\mathrm{WLr}}$, which is $\mathrm{G} 3 p$ extended by rule

$$
\frac{\square \Pi, \Gamma \Rightarrow \varphi}{\Lambda, \square \Pi, \square \Gamma \Rightarrow \square \varphi, \Theta} \mathcal{R}_{\mathrm{WLr}},
$$


cannot be a calculus for K4. And because $\mathrm{G} 3 p+\mathcal{R}_{\mathrm{WLr}}$ would be equivalent to one of the standard calculi for K4, were it closed under contraction, the calculus G3p $+\mathcal{R}_{\mathrm{WLr}}$ cannot be closed under contraction. Thus it is a calculus for a substructural logic. This in contrast to rules that are not WL-rules or RE-rules: extending G3p by such rules preserves the admissibility of contraction and weakening.

Acknowledgements I thank an excellent anonymous referee for the detailed comments on earlier drafts of this paper. Support by the Netherlands Organisation for Scientific Research under Grant 639.032.918 is gratefully acknowledged.

Open Access This article is distributed under the terms of the Creative Commons Attribution 4.0 International License (http://creativecommons.org/licenses/by/4.0/), which permits unrestricted use, distribution, and reproduction in any medium, provided you give appropriate credit to the original author(s) and the source, provide a link to the Creative Commons license, and indicate if changes were made.

\section{References}

1. Bílková, M.: Uniform interpolation and propositional quantifiers in modal logics. Stud. Log. 85(1), 259-271 (2007)

2. Dershowitz, N., Manna, Z.: Proving termination with multiset orderings. Commun. ACM 22, 465-476 (1979)

3. Ghilardi, S., Zawadowski, M.: Undefinability of propositional quantifiers in the modal system S4. Stud. Log. 55, 259-271 (1995)

4. Ghilardi, S., Zawadowski, M.: Sheaves, Games, and Model Completions: A Categorical Approach to Nonclassical Propositional Logics. Trends in Logic (Book 14). Springer, Berlin (2002)

5. Maxsimova, L.L.: Craig's theorem in superintuitionistic logics and amalgamated varieties of pseudoBoolean algebras. Algebra Log. 16(6), 643-681 (1977)

6. Pitts, A.: On an interpretation of second order quantification in first order intuitionistic propositional logic. J. Symb. Log. 57(1), 33-52 (1992)

7. Shavrukov, V.Y.: Subalgebras of diagonalizable algebras of theories containing arithmetic. Dissertationes Mathematicae, vol. 323. Instytut Matematyczny Polskiej Akademi Nauk (Warszawa) (1993)

8. Troelstra, A.S., Schwichtenberg, H.: Basic Proof Theory. Cambridge Tracts in Theoretical Computer Science, vol. 43. Cambridge University Press, Cambridge (1996)

9. Visser, A.: Bisimulations, Model Descriptions and Propositional Quantifiers. Logic Group Preprint Series, vol. 161. Utrecht University (1996)

10. Visser, A.: Uniform interpolation and layered bisimulation. Lect. Notes Log. 6, 139-164 (1996) 